This is a non-peer-reviewed manuscript which has been submitted to Journal of Geophysical Research — Solid Earth 


\title{
Experimental multiblast craters and ejecta seismo-acoustics, jet characteristics, craters, and ejecta deposits and implications for volcanic explosions
}

\author{
Ingo Sonder ${ }^{1}$, Alison Graettinger ${ }^{2}$, Tracianne B. Neilsen ${ }^{3}$, Robin S. Matoza ${ }^{4}$, \\ Jacopo Taddeucci ${ }^{5}$, Julie Oppenheimer ${ }^{6}$, Einat Lev ${ }^{6}$, Kae Tsunematsu ${ }^{7}$, \\ Greg Waite $^{8}$, Greg A. Valentine ${ }^{1}$ \\ ${ }^{1}$ Center for Geohazards Studies, University at Buffalo, Buffalo, NY, USA \\ ${ }^{2}$ Department of Earth \& Environmental Sciences, University of Missouri Kansas City, Kansas City, MO, \\ USA \\ ${ }^{3}$ Department of Physics and Astronomy, Brigham Young University, Provo, UT, USA \\ ${ }^{4}$ Department of Earth Science and Earth Research Institute, University of California, Santa Barbara, CA, \\ USA \\ ${ }^{5}$ Istituto Nazionale di Geofisica e Vulcanologia, Rome, Italy \\ ${ }^{6}$ Lamont Doherty Earth Observatory, Columbia University, Palisades, NY, USA \\ ${ }^{8}$ Geological and Mining Engineering and Sciences, Michigan Tech, Houghton, MI, USA
}

\section{Key Points:}

- Airborne energy of an underground blast decays exponentially with scaled depth and is in agreement with previous measurements.

- Multiple subsurface explosions, properly timed, can break the surface from scaled depths previously thought to be contained in the ground.

- Crater sizes correlate with measured seismo-acoustic and high-frequency atmospheric signals. 


\begin{abstract}
Blasting experiments were performed that investigate multiple explosions that occur in quick succession in the ground and their effects on host material and atmosphere. Such processes are known to occur during volcanic eruptions at various depths, lateral locations, and energies. The experiments follow a multi-instrument approach in order to observe phenomena in the atmosphere and in the ground, and measure the respective energy partitioning. The experiments show significant coupling of atmospheric (acoustic)and ground (seismic) signal over a large range of (scaled) distances (30-330 $\mathrm{m}, 1-10 \mathrm{~m} \mathrm{~J}^{-1 / 3}$ ). The distribution of ejected material strongly depends on the sequence of how the explosions occur. The overall crater sizes are in the expected range of a maximum size for many explosions and a minimum for one explosion at a given lateral location. The experiments also show that peak atmospheric over-pressure decays exponentially with scaled depth at a rate of $\bar{d}_{0}=6.47 \times 10^{-4} \mathrm{~m} \mathrm{~J}^{-1 / 3}$; at a scaled explosion depth of $4 \times 10^{3} \mathrm{~m} \mathrm{~J}^{-1 / 3}$ ca. $1 \%$ of the blast energy is responsible for the formation of the atmospheric pressure pulse; at a more shallow scaled depth of $2.75 \times 10^{-3} \mathrm{~m} \mathrm{~J}^{-1 / 3}$ this ratio lies at ca. 5.57.5\%. A first order consideration of seismic energy estimates the sum of radiated airborne and seismic energy to be up to $20 \%$ of blast energy.
\end{abstract}

\title{
Plain Language Summary
}

Blasting experiments using six successive explosions were performed in four different geometrical setups (linear and triangular). The experiments were monitored by geophysical equipment which allows to measure explosive energy, and how much of that energy goes to the surface. The experiments help to understand volcanic and other subsurface explosive processes. Exact measurements of the resulting craters, together with known explosive energies allow the interpretation of real volcanic craters. The experimental results show initial time developments of crater sizes, which occurs on the order of one second for crater sizes of the order of one meter. Up to $8 \%$ of the explosion's energy was detected as airborne signal. Up to $20 \%$ of the explosion's energy was detected as seismic (elastic) energy in the ground. 


\section{Introduction}

Volcanic activity causes subsurface explosions at various depths that can have severe consequences for its environment. Explosions can have several causes, but it is possible to evaluate some of their aspects independent from their cause. A sudden, large pressure change propagates at supersonic speed for a certain distance in a medium such as host rock, magma or atmosphere, causing deformation in elastic, plastic and brittle regimes (e.g. Schnurr et al., 2020; Kim \& Rodgers, 2016; Bowman et al., 2014; Fee et al., 2013; Taylor et al., 2010; Grady, 1996). Shallow explosions fragment and eject magma, host material or both into the atmosphere and pose danger to the surroundings. Deeper explosions (for a given energy release) may be fully contained in the subsurface (Valentine et al., 2014). In case of a subsurface explosion parts of the energy involved will end up in the atmosphere, while some of it will remain in the ground. In volcanic settings explosions may occur as individual events or in rapid succession, at various depths and lateral locations. Characterizing the transition from a fully contained process to near surface is important to estimate the hazards to surroundings and understand some principle mechanisms of the explosion process. Many mechanisms can cause volcanic explosions (Houghton, 2015), but some effects on the surroundings are common to all explosive source mechanisms. For example, all explosive processes mix host material, and shallow explosions eject significant amounts of hot material (Graettinger et al., 2015). Subsurface explosions produce crater structures, that are characteristic for the blast process's energy and location (Valentine et al., 2014).

In natural settings, explosive volcanic blasts and processes are often monitored using multiple techniques, including seismic and infrasound observation and video recordings at normal and high speeds (Gaudin et al., 2016; Matoza et al., 2019). Seismoacoustics aims to relate signatures of observed seismic and infrasound waveforms to the source processes generating them. A more controlled process than the poorly constrained natural signals, with known source parameters can help to constrain uncertainties and enable scalability of models.

An explosion - a sudden, rapid change of a material's volume that it imposes on its surroundings - forces that medium to rapidly compress such that the resulting pressure change does not propagate with the same speed as a smaller pressure change would which is described within the linear acoustic approximation. Larger pressure changes cause adiabatic heating in air which locally increases the propagation speed and can lead to dramatic steepening of an initially smooth pressure wave into a discontinuity - a shock (Garcés et al., 2013; Muhlestein et al., 2012; Crighton \& Scott, 1979). In an isentropic approximation (reversible process at constant entropy) a shock pulse has characteristic properties such as amplitude and duration that scale with the explosion's energy and the density of the medium in which the pulse travels (Kinney \& Graham, 1985).

Scaling properties enable the establishment of phenomenological regimes that depend on scaled parameters, such as a scaled length. For example, for the depth $d$ of a subsurface explosion, a scaled depth can be defined by

$$
\bar{d}=\frac{d}{E_{\mathrm{b}}^{1 / 3}},
$$

where $E_{\mathrm{b}}$ is the blast's energy (Holsapple \& Schmidt, 1980; Sonder et al., 2015). Using this method blasts of any energy may be categorized into deep, intermediate and shallow blasts. Deep blasts are contained in the ground and do not eject material $\left(\bar{d} \gtrsim 8 \times 10^{-3} \mathrm{~m} \mathrm{~J}^{-1 / 3}\right)$. The host material's weight and strength are large enough to "contain" the blasts. Energy is dissipated by friction and anelastic alteration, or transported elastically as seismic waves. At intermediate scaled depths $\left(\bar{d} \simeq 4 \times 10^{-3} \mathrm{~m} \mathrm{~J}^{-1 / 3}\right)$, material is excavated efficiently, which results in the largest craters. Shallow blasts $\left(\bar{d}<4 \times 10^{-3} \mathrm{~m} \mathrm{~J}^{-1 / 3}\right)$ create a smaller crater. Larger parts of $E_{\mathrm{b}}$ couple with the atmosphere and fewer with the 
host, resulting in a large atmospheric pressure pulse. These regimes are backed up by extensive studies from military and mining research (Holsapple \& Schmidt, 1980; Lee \& Mazzola, 1989; Ehrgott et al., 2011; Dillon, 1972; Qiu et al., 2018), as well as research motivated by volcanology (Ambrosini et al., 2002; Sato \& Taniguchi, 1997; Goto et al., 2001; Valentine et al., 2012; Sonder et al., 2015; Ross et al., 2013). Two lengths which scale with the $1 / 3$ power of $E_{\mathrm{b}}$ and which differ by a factor 2 , for example two crater radii created by two single subsurface blasts, were caused by blast energies which differed by a factor $2^{3}=8$.

Similar phenomenological regimes exist for a blast wave propagating in air. The distance from explosion source, $r$, may be scaled by blast energy and air density $\rho$

$$
\bar{r}=\frac{\rho r}{\rho_{0} E_{\mathrm{b}}^{1 / 3}} .
$$

The reference density $\rho_{0}$ is a value known from a case for which the scaled distance is known. Similar to $\bar{d}, \bar{r}$ may be used to categorize an observation distance into far $(\bar{r} \gtrsim$ $\left.6 \times 10^{-2} \mathrm{~m} \mathrm{~J}^{-1 / 3}\right)$, in which the peak pressure drops with $\bar{r}^{-1}$, intermediate $\left(\bar{r} \simeq 6 \times 10^{-3} \mathrm{~m} \mathrm{~J}^{-1 / 3}\right)$, or near $\left(\bar{r} \lesssim 10^{-3} \mathrm{~m} \mathrm{~J}^{-1 / 3}\right)$, (Kinney and Graham (1985)).

Less studied, from a volcanological perspective, is the effects of scaled depth on monitoring signals such as seismic, acoustic, and infrasound, particularly in cases involving multiple explosions occurring in rapid succession. Crater structures and ejecta products of such blasts are analyzed, and allow to connect their geometries and stratigraphy to energy, explosion locations and sequencing. These field findings also reveal the complexities of the natural processes, which limit the straight forward application of simple explosion models (Taddeucci et al., 2010). Some factors controlling the dynamic behavior and energy scaling have a common base with other applications of explosives in the fields of military or mining research (Ambrosini \& Luccioni, 2006; Qiu et al., 2018). Such applications allow the scaling of lengths with a blast's energy, and use the depth below the surface to quantify its confinement. The scaling relationships were found experimentally, and while in detail the phenomena associated with a subsurface explosion depends on factors such as host material strength, rough phenomenological regimes can be identified that are primarily related to energy and depth combinations. Energy scaling was experimentally verified across length scales ranging from $10^{-2} \mathrm{~m}$ to $10^{3} \mathrm{~m}$, and energies from $10^{3} \mathrm{~J}$ to $10^{15} \mathrm{~J}$ (Strange et al., 1960; Vortman, 1968; Sato \& Taniguchi, 1997). Energies of most volcanic eruptions fall into this range (Valentine et al., 2014), motivating either direct applicability of the methods or a version adapted to volcanic activity.

Here we report results of experiments that focus on the effects of multiple explosions, closely spaced and timed, on ejecta, crater morphology, and geophysical signals. Such explosions show different behavior depending on the state of topography and host conditions at time of explosion. Both are varying rapidly, which causes ejecta jets to become asymmetric (Figure 1, supporting video S1-S4), and can be observed on volcanic scale (Voight, 1981). A volcanic explosive source was replaced by time- and energy-constrained chemical explosions. Previous experimental studies showed that this approach has important implications for field-scale analysis and interpretation (Sato \& Taniguchi, 1997; Goto et al., 2001; Graettinger et al., 2014; Bowman et al., 2014; Valentine et al., 2014, 2015; Sonder et al., 2015; Graettinger et al., 2015; Macorps et al., 2016; Graettinger, Valentine, \& Sonder, 2015). In these previous experiments explosive charges were detonated separately, and the effects of each single detonation on the surface morphology and ejected material were studied before detonating the next charge. While the approach is relevant to many volcanic settings, observation shows that during explosive eruptions many explosions can occur closely spaced in time (Matoza et al., 2014; Park et al., 2021) or simultaneously, superposing their tephra jets, to create one single cumulative eruption column (Dürig, Gudmundsson, \& Dellino, 2015). Our study tests whether the results of pre- 

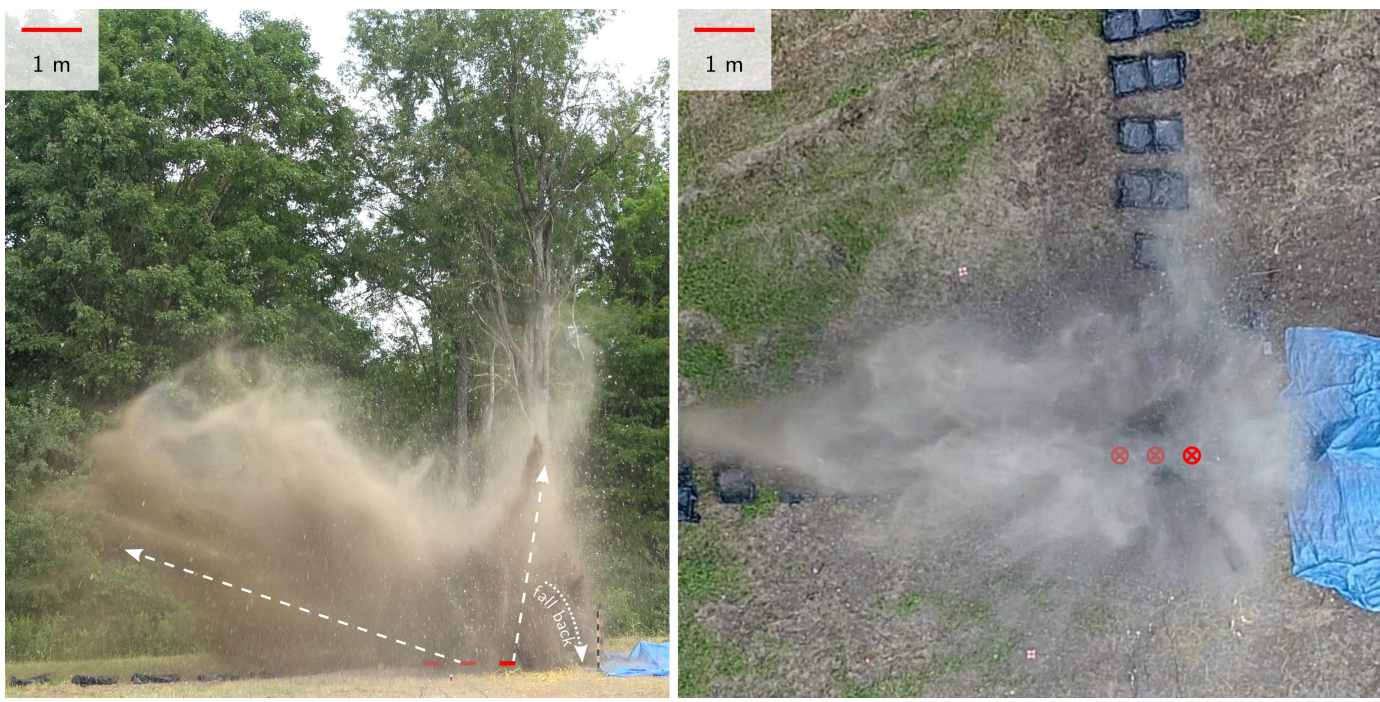

Figure 1: Side- and top view of a typical asymmetric ejecta jet created by the detonation sequences. Red markers show surface of charge locations. The example shows the jet of the third detonation in the "pad 1" configuration (See also supporting video S1).

vious experiments with separate blasts can be extended to those with blasts in rapid succession and with lateral and vertical migration.

\section{Methods and Experimental Setup}

For each of the experiments reported here six charges were buried and detonated in test pads which were filled with unconsolidated granular material. The setup roughly follows previous studies on craters, each of which was created by more than one explosion ("multiblast craters") in which charges were detonated, and their blasts studied one at a time (Valentine et al., 2012; Graettinger et al., 2014; Sonder et al., 2015). The explosive material was Pentex ${ }^{\mathrm{TM}}$, which is a proprietary compound material with major components including trinitrotoluene (TNT) and pentaerythritol (PETN). It has a specific energy of $4.85 \times 10^{6} \mathrm{~J} \mathrm{~kg}^{-1}$; each charge had a mass of $90 \mathrm{~g}$ which corresponds to an energy of $4.37 \times 10^{5} \mathrm{~J}$. The six charges were detonated in a timed sequence of $0.5 \mathrm{~s}$ between each detonation. Accuracy of detonation timing was better than $10^{-3} \mathrm{~s}$. This timing was selected to ensure that the ejecta jet of each blast interacted with that of the preceding blast. Two plan-view configurations were set up; one with three charge epicenters in a line; another with three epicenters corresponding to the apexes of a triangle. Charges were arranged vertically on top of one another, at two depths, $30 \mathrm{~cm}$ and $60 \mathrm{~cm}$ (Figure 2). At the given blast energy $30 \mathrm{~cm}$ corresponds to a scaled explosion depth of $3.95 \times 10^{-3} \mathrm{~m} \mathrm{~J}^{-1 / 3}$, a value very close to optimum excavation conditions. Horizontal spacing was chosen, such that the horizontal neighbor charge location would be within the footprint of a single blast at optimum depth, but close to its border. At pads 1 and 3 the upper charges were detonated in sequence, followed by the three lower charges. At pads 2 and 4 , charges beneath each epicenter were detonated in a sequence of shallow-first and deeper-second (Figure 3$)$.

The blast sequences were monitored by high-speed and normal speed video cameras. A set of six cameras was arranged in a hemicycle, at a distance between $20-30 \mathrm{~m}$ to accurately capture directions of ejected materials. Drone-based video was recorded 


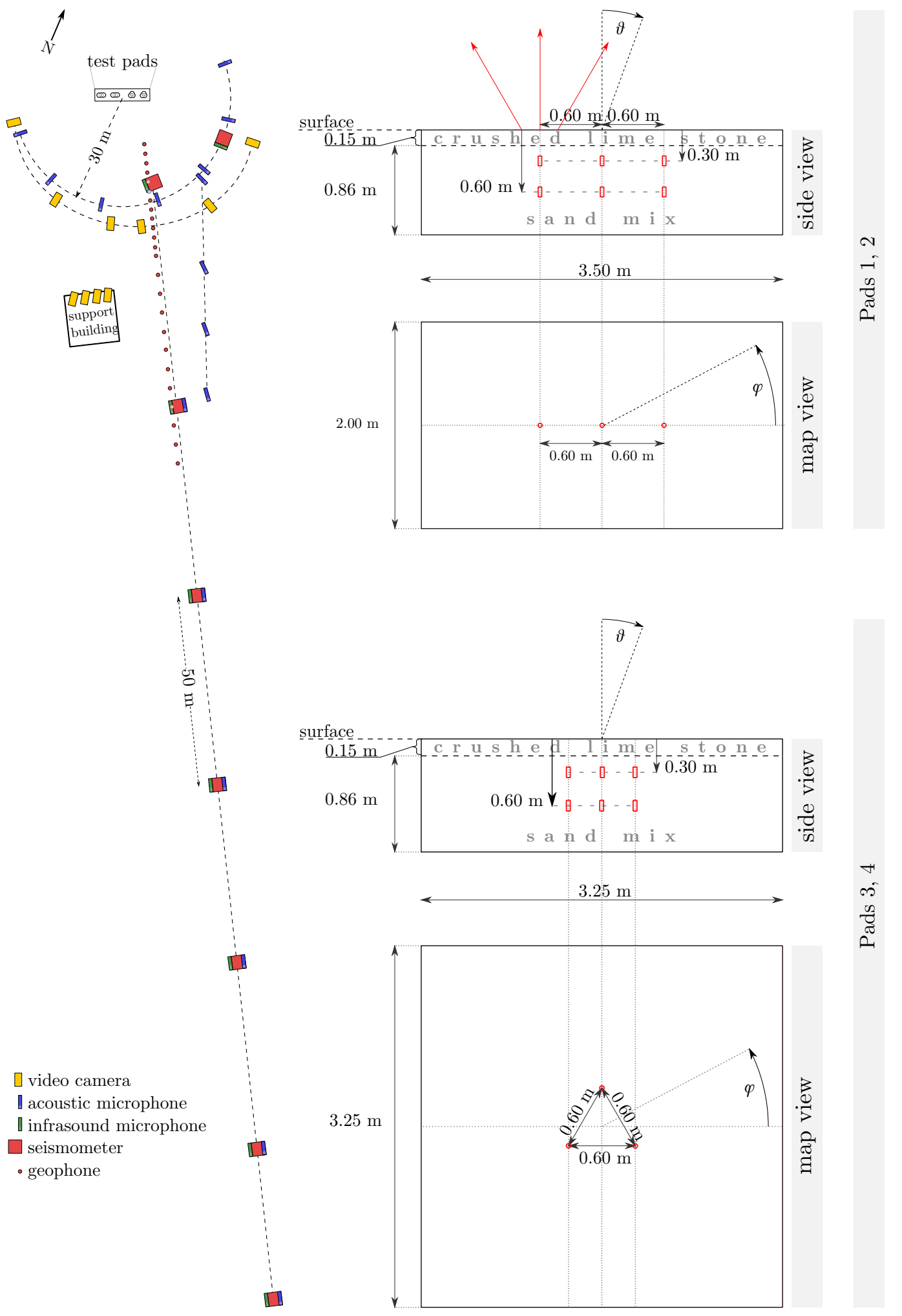

186

Figure 2: (Caption on next page) 
Figure 2: Multi-sensor stations were placed in a radial line every $50 \mathrm{~m}$ starting at $30 \mathrm{~m}$ distance from the test pads. Each station included compact broadband seismic and infrasonic sensors as well as broadband ("acoustic") microphones. Acoustic microphones were placed in a $30 \mathrm{~m}$ radius semicircle around the center of the test pads. Another set of microphones was placed in a radial line from the test pads ranging from $30 \mathrm{~m}$ to $80 \mathrm{~m}$ distance. 12 geophones were placed every $2.5 \mathrm{~m}$ starting at $12 \mathrm{~m}$ distance from the pads center, and 11 more along the same direction every $5 \mathrm{~m}$ following that. The last geophone had a distance of $99.5 \mathrm{~m}$ from the pads center. Six identical cameras recorded the experiments also in an arc of about $30 \mathrm{~m}$ distance. Other cameras recorded from a $50 \mathrm{~m}$ distance location.

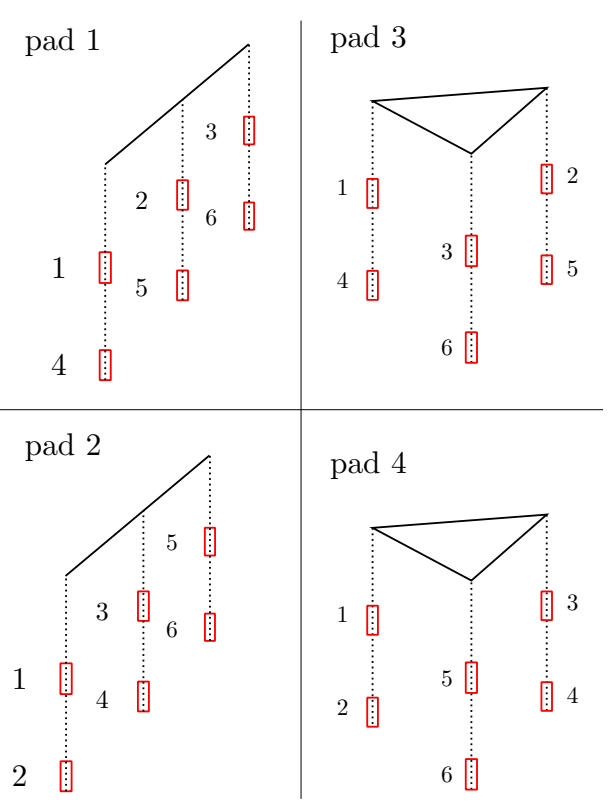

Figure 3: Firing sequence of the four test pads. Numbers indicate the position of the firing sequence. Charges were fired in one after another in $0.5 \mathrm{~s}$ intervals. For any number $i$ between 1 and 6 the corresponding charge was fired $(i-1) \cdot 0.5 \mathrm{~s}$ after the first charge. In pads 1 and 3 the upper charges (buried at $30 \mathrm{~cm}$ depth) were the first three to be fired, before the lower level (buried at $60 \mathrm{~cm}$ depth) was fired in the same lateral sequence as the upper ones. In pads 2 and 4 charge pairs located at same horizontal location were fired consecutively (upper level $0.5 \mathrm{~s}$ before lower level).

to determine lateral jet directions and material motion. High-speed cameras recorded at 300,500 and $5000 \mathrm{fps}$.

Seismo-acoustic records were made using a combination of seismometers, geophones, infrasound-microphones ("infrasound sensors") and higher frequency broadband microphones ("acoustic microphones"). The deployed seismometers and infrasound sensors fit into the SEED broadband category (band code "C", Ahern \& Dost, 2012). Seismometers and infrasound sensors were recorded at $400 \mathrm{~Hz}$ or $500 \mathrm{~Hz}$. Deployed infrasound sensors had a flat frequency response between $3 \times 10^{-2} \mathrm{~Hz}$ and Nyquist frequency. Two types of the acoustic microphones were used, with linear $( \pm 2 \mathrm{~dB})$ response from $3.15 \mathrm{~Hz}$ to $20 \mathrm{kHz}$ and $4 \mathrm{~Hz}$ to $80 \mathrm{kHz}$ (Table 1). Despite the short hand "acoustic microphones" these sensors range far into the ultrasonic range. Recordings in this frequency range are very rare 
for volcano seismo-acoustics or not available at all. High-frequency recordings typically end around $10 \mathrm{kHz}$ (Taddeucci et al., 2021).

From these sensors seismo-acoustic measurement stations were assembled for specific purposes. Station type (a) was dedicated to measure the radial decay of airborneand ground based blast signals. For each of the type (a) statios a 3-component seismometer, an infrasound microphone and two acoustic microphones were used. The seismometer was placed $1 \mathrm{~m}$ below-, the infrasound sensor just below the surface. The microphones were mounted $4 \mathrm{~m}$ above ground, pointing towards the blast source, and just above ground, pointing downwards. Seven type (a) stations were placed every $50 \mathrm{~m}$ in a radial line, starting at $30 \mathrm{~m}$ distance from the test pads center, so that the last station was at $380 \mathrm{~m}$ distance (Figure 2). Station type (b) was dedicated to the depth dependency of blast signals. One station was assembled which consisted of three 3-component seismometers, placed $132 \mathrm{~cm}, 75 \mathrm{~cm}$ and $18 \mathrm{~cm}$ below the surface, and one infrasound sensor, placed just below the surface. Station (b) had a distance of $30 \mathrm{~m}$ from the blast pads center (Figure 2). Station type (c) was dedicated to measure the angular dependency of the airborne signals. For each of them two acoustic microphones were placed $2.44 \mathrm{~m}$ and $1.22 \mathrm{~m}$ above ground. Type (c) stations were placed in a $30 \mathrm{~m}$ radius semi-circle around the center of the blast pads. Angles range from $0^{\circ}$ to $180^{\circ}$ and were arranged so that the $90^{\circ}$ station was also the start of the type (a) radial line (Figure 2). Seismo-acoustic setup also included a line of 23 geophones to record ground speeds at $12 \mathrm{~m}-100 \mathrm{~m}$ distance along the type (a) radial line.

Ejected material was collected in two box arrays, separated at an angle $>45^{\circ}$ to collect material from $2.5-13.5 \mathrm{~m}$ from the charge assembly's center. The sample arrays were re-positioned for each experiment, so that they were always centered around an explosion site. One array was typically at an angle $\phi=90^{\circ}$. The other array had different orientations for each pad, because other equipment and arrangements restricted the available space (Figure 5).

After the charges had detonated and ejecta jets had dissipated, photographs of the produced compound craters were taken for photogrammetry (structure from motion) analysis. Photographs were taken using (a) the same UAVs that also recorded blast videos, and (b) using a standard SLR camera, operated by a (ground-based) person. A subset of the photographs was the base for digital elevation models (DEMs) that were created using the commercial photogrammetry software Metashape ${ }^{\mathrm{TM}}$, generally following previous experiments (Graettinger, Valentine, \& Sonder, 2015). The resulting DEMs have a spatial resolution between $1 \mathrm{~cm}$ and $1.5 \mathrm{~cm}$ for pads $1-3$, and $2.5 \mathrm{~cm}$ for pad 4 . All crater profiles- and sizes presented below are based on these elevation models. 
Table 1: Sensor setup of the three seismo-acoustic station types.

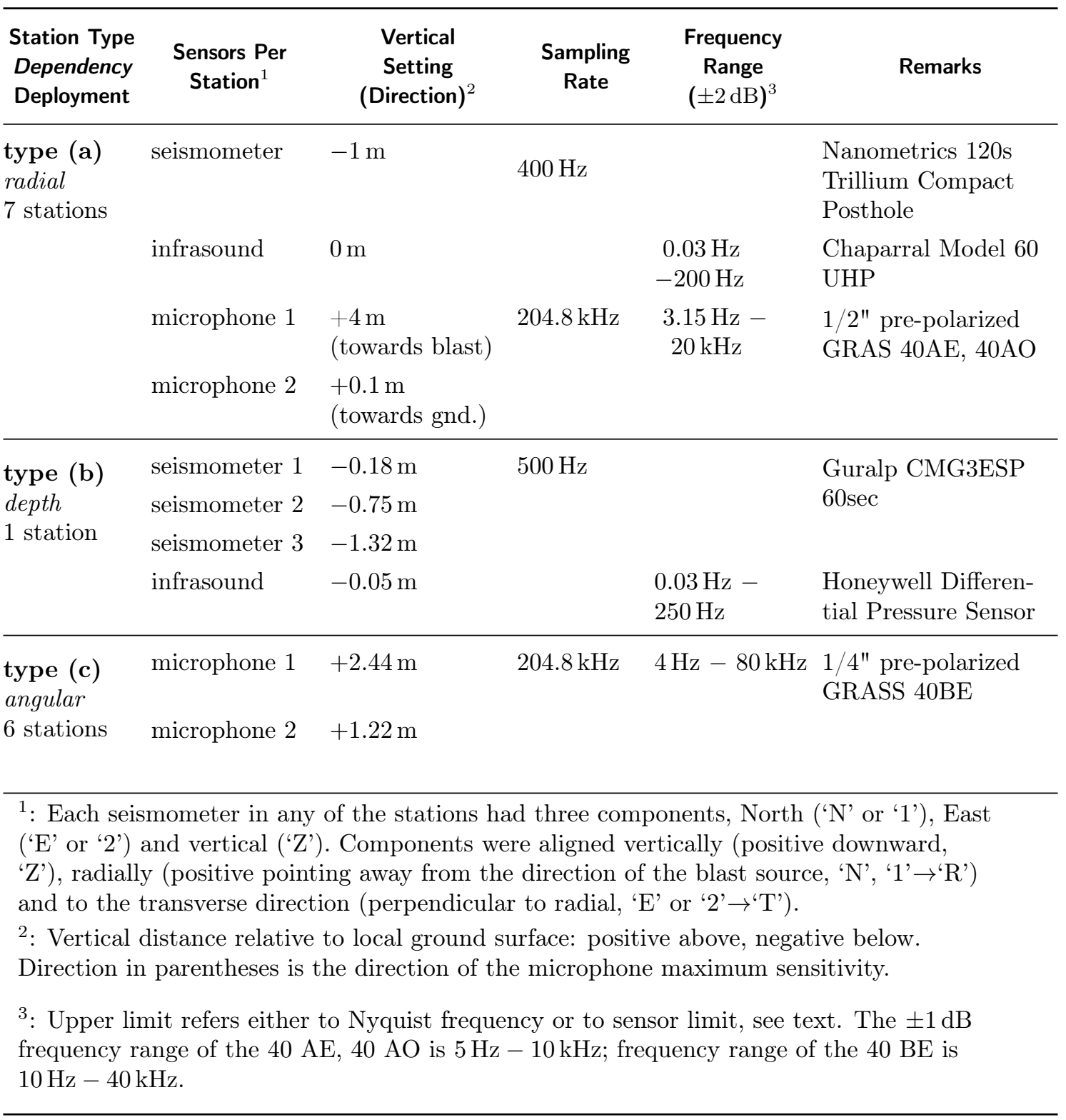




\section{Observations and Results}

\subsection{Qualitative Observations}

For all pads, the initial blast transported the greatest mass of material. From the main observation direction this charge was located at the top-left end of the linear setups of pads 1 and 2, and at the top-rear corner of the triangular setups of pads 3 and 4 . Size and speed of these initial blasts (jets) were comparable to previously conducted experiments (Valentine et al., 2012). Ejecta jets of the quieter blasts showed similar thinning behavior as was observed in previous experiments for blasts under pre-existing cratertopography (Ross et al., 2013; Graettinger et al., 2015). Some jets had a main direction that was not vertical, but had a certain direction towards the main (temporally changing) crater void showing similarities with previously conducted off-center blast experiments (Valentine et al., 2015). For pads 2 and 4, for which the lower charges were fired only $0.5 \mathrm{~s}$ after the upper charge (at same lateral location), the perceived loudness (not measured amplitude) of these lower charges was significantly larger compared to the previous optimum depth blast. In contrast, for pads 1 and 3, for which lower charges were fired $1.5 \mathrm{~s}$ after the upper charge at same lateral location, the blast noise was significantly muffled (Table 2).

\subsection{Jets, Craters and Ejecta}

Unlike past experiments in which a crater was analyzed after each individual blast, the timing of these multiblast experiments only allows for inspection of the final crater and ejecta. This crater is the cumulative product of six blasts that migrate vertically and laterally through the host. The blast sequences in pads 1 and 2 created craters elongated along the axis of the charges. The final craters of the triangular blast sequences (pads 3 and 4) were more round, with some visibility of single-charge crater outlines in the triangle's corners (Figure 4).

The deepest points of the pad 1 and pad 2 craters were located between the central and right charge positions in the $x$-direction, and in close proximity to the symmetry line along the charges in $y$-direction. The lower right charge was always the last to detonate. The crater profiles preserved a stepped floor centered over the final charge (Figure 4). The ejecta showed a prominent ray (ridge of material) that extended from the final charge location out of the crater in the direction of elongation $\left(\varphi=180^{\circ}\right)$. Parts of the ray could be traced more than $10 \mathrm{~m}$ from the crater. For pad 1, one of the ejecta sample arrays was in line with this ray (supplementary video S1); in this direction the ejected mass per area was a factor $\simeq 10$ higher compared to the material collected by the array perpendicular to the charge line (Figure 5). Also, mass distribution is better described by an exponential distribution in the $\varphi=180^{\circ}$-direction compared to the $90^{\circ}$ direction which is better approximated by a power law. Isolated pieces of shallow-sourced gravel from pads 1 and 2 were observed further from the charges; one of them over $30 \mathrm{~m}$ away from pad 2 , in the $\varphi=180^{\circ}$-direction.

The asymmetry of ejecta distribution around the linear charge array is similar to what was observed in previous off-center multiblast configurations with temporally well separated charge detonations (Valentine et al., 2015). However, in those experiments a steep ejecta ring was formed on the side of the crater opposite to the direction of jet inclination (Graettinger, Valentine, \& Sonder, 2015). This steep ejecta rim was not observed in the here presented, overlapping blast sequences.

The triangular blast sequences of pads 3 and 4 produced more equant crater shapes resembling blurred circles around the triangular blast centers (Figure 4). Compared to the linear setups the deepest points of the craters were located laterally closer to the centroid and had a larger distance to the last blast's center. The pad 3 crater had a low point between the first and second (lateral) blast locations. Pad 4 had the low point close to 
Table 2: Qualitative comparison of blast experiment configuration and resulting noise and direction. The "left" and "right" labels refer to the jet directions as seen from the main observation location. Polar- and inclination angles are also illustrated in Figure 2.

\begin{tabular}{|c|c|c|c|c|c|c|c|}
\hline pad & blast & depth & $\begin{array}{l}\text { delay after } \\
1^{\text {st }} \mathrm{chrg} \text {. }\end{array}$ & $\begin{array}{l}\text { delay after } \\
\text { corresp. chrg. }{ }^{1}\end{array}$ & $\begin{array}{l}\text { perceived } \\
\text { loudness }\end{array}$ & $\begin{array}{c}\text { Incli- } \\
\text { nation }\end{array}(\theta)$ & $\begin{array}{l}\text { approx. polar } \\
\text { angle }(\varphi)^{2}\end{array}$ \\
\hline \multirow[t]{6}{*}{1} & 1 & $30 \mathrm{~cm}$ & $0 \mathrm{~s}$ & $0 \mathrm{~s}$ & medium & none & - \\
\hline & 2 & $30 \mathrm{~cm}$ & $0.5 \mathrm{~s}$ & $0 \mathrm{~s}$ & medium & $>30^{\circ}$ & $180^{\circ}$ (left) \\
\hline & 3 & $30 \mathrm{~cm}$ & $1 \mathrm{~s}$ & $0 \mathrm{~s}$ & medium & $>30^{\circ}$ & $180^{\circ}$ (left) \\
\hline & 4 & $60 \mathrm{~cm}$ & $1.5 \mathrm{~s}$ & $1.5 \mathrm{~s}$ & muffled & none & $0^{\circ}(-)$ \\
\hline & 5 & $60 \mathrm{~cm}$ & $2 \mathrm{~s}$ & $1.5 \mathrm{~s}$ & muffled & $>30^{\circ}$ & $180^{\circ}$ (left) \\
\hline & 6 & $60 \mathrm{~cm}$ & $2.5 \mathrm{~s}$ & $1.5 \mathrm{~s}$ & muffled & $>30^{\circ}$ & $180^{\circ}$ (left) \\
\hline \multirow[t]{6}{*}{2} & 1 & $30 \mathrm{~cm}$ & $0 \mathrm{~s}$ & $0 \mathrm{~s}$ & medium & none & - \\
\hline & 2 & $60 \mathrm{~cm}$ & $0.5 \mathrm{~s}$ & $0.5 \mathrm{~s}$ & loud & none & - \\
\hline & 3 & $30 \mathrm{~cm}$ & $1 \mathrm{~s}$ & $0 \mathrm{~s}$ & medium & $>30^{\circ}$ & $180^{\circ}$ (left) \\
\hline & 4 & $60 \mathrm{~cm}$ & $1.5 \mathrm{~s}$ & $0.5 \mathrm{~s}$ & loud & $>30^{\circ}$ & $180^{\circ}$ (left) \\
\hline & 5 & $30 \mathrm{~cm}$ & $2 \mathrm{~s}$ & $0 \mathrm{~s}$ & muffled & $>30^{\circ}$ & $180^{\circ}$ (left) \\
\hline & 6 & $60 \mathrm{~cm}$ & $2.5 \mathrm{~s}$ & $0.5 \mathrm{~s}$ & loud & $\lesssim 20^{\circ}$ & $95^{\circ}$ (left) \\
\hline \multirow[t]{6}{*}{3} & 1 & $30 \mathrm{~cm}$ & $0 \mathrm{~s}$ & $0 \mathrm{~s}$ & medium & none & - \\
\hline & 2 & $30 \mathrm{~cm}$ & $0.5 \mathrm{~s}$ & $0 \mathrm{~s}$ & medium & medium & $135^{\circ}$ (left) \\
\hline & 3 & $30 \mathrm{~cm}$ & $1 \mathrm{~s}$ & $0 \mathrm{~s}$ & medium & large & $30^{\circ}$ (right) \\
\hline & 4 & $60 \mathrm{~cm}$ & $1.5 \mathrm{~s}$ & $1.5 \mathrm{~s}$ & muffled & low & $270^{\circ}(-)$ \\
\hline & 5 & $60 \mathrm{~cm}$ & $2 \mathrm{~s}$ & $1.5 \mathrm{~s}$ & muffled & medium & $150^{\circ}$ (left) \\
\hline & 6 & $60 \mathrm{~cm}$ & $2.5 \mathrm{~s}$ & $1.5 \mathrm{~s}$ & muffled & large & $30^{\circ}$ (right) \\
\hline \multirow[t]{6}{*}{4} & 1 & $30 \mathrm{~cm}$ & $0 \mathrm{~s}$ & $0 \mathrm{~s}$ & medium & none & - \\
\hline & 2 & $60 \mathrm{~cm}$ & $0.5 \mathrm{~s}$ & $0.5 \mathrm{~s}$ & loud & none & - \\
\hline & 3 & $30 \mathrm{~cm}$ & $1 \mathrm{~s}$ & $0 \mathrm{~s}$ & medium & medium & $135^{\circ}$ (left) \\
\hline & 4 & $60 \mathrm{~cm}$ & $1.5 \mathrm{~s}$ & $0.5 \mathrm{~s}$ & loud & low & $135^{\circ}$ (left) \\
\hline & 5 & $30 \mathrm{~cm}$ & $2 \mathrm{~s}$ & $0 \mathrm{~s}$ & medium & medium & $<30^{\circ}$ (right) \\
\hline & 6 & $60 \mathrm{~cm}$ & $2.5 \mathrm{~s}$ & $0.5 \mathrm{~s}$ & loud & low & $<30^{\circ}$ (right) \\
\hline
\end{tabular}

1: Delay of the lower charges, relative to the upper charge at same lateral location (cf. Figure 3).

2: Polar angle is counted counter clock wise, and $0^{\circ}$ along the axis parallel to the charge lines of pads 1 and 2, pointing to the right as seen from main observation direction. 

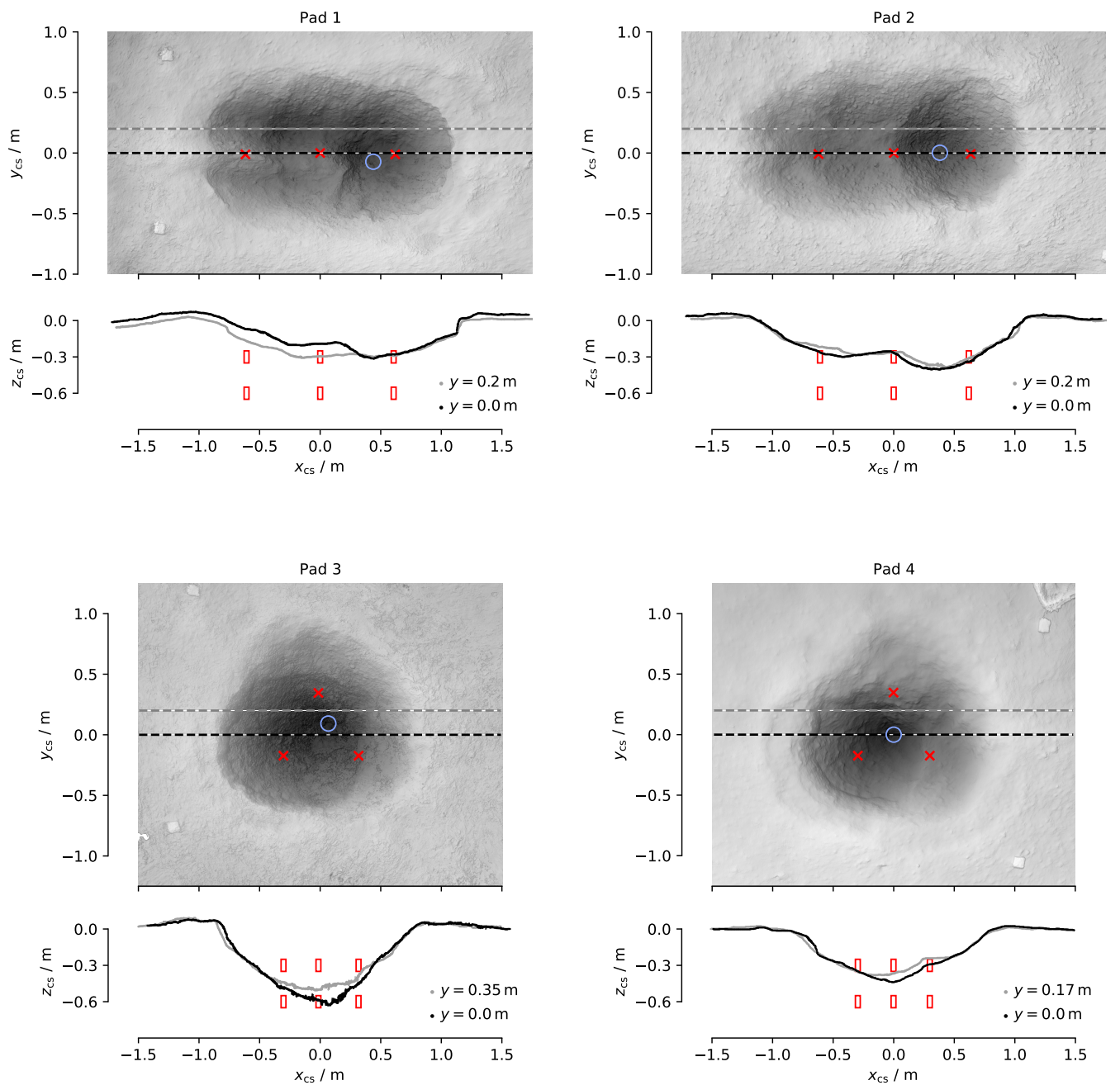

Figure 4: Map view and selected crater profiles of the crater structures. Red crosses and boxes mark the locations of explosive charges, blue circles show the deepest points of the craters. The pre-blast surface was at $z_{\mathrm{cs}}=0$. The linear charge arrangements (pads 1 and 2) created a stepped profile that reflect the blast history to some extent. Their deepest point was about $30 \mathrm{~cm}$, the upper charge depth. Sequences shot in the triangular geometries (pads 3 and 4) excavated significant amounts of material from below $30 \mathrm{~cm}$. 
its centroid. Both of the craters had shallow slopes near the crater rim, and steeper slopes closer to the center. Ejecta were concentrated in three main directions for pad 3, and two for pad 4. Compared to the linear charge setups, the observed ejecta concentrations of the triangular sequences were less pronounced. The ejecta concentrations originate from one vertex of the charge configuration to bisect the opposite side of the triangle (supporting video S3). The pad 3 sequence had ejecta concentrations correlating to all three lateral charge positions. In the pad 4 sequence ejecta rays only correlated to blasts 3,4 $\left(\varphi \simeq 150^{\circ}\right)$ and $5,6\left(\varphi \simeq 30^{\circ}\right)$, since the first two blasts occurred in an effectively radially symmetric setting (blast 1 under flat topography, blast 2 under an approximately radially symmetric transient cavity). 

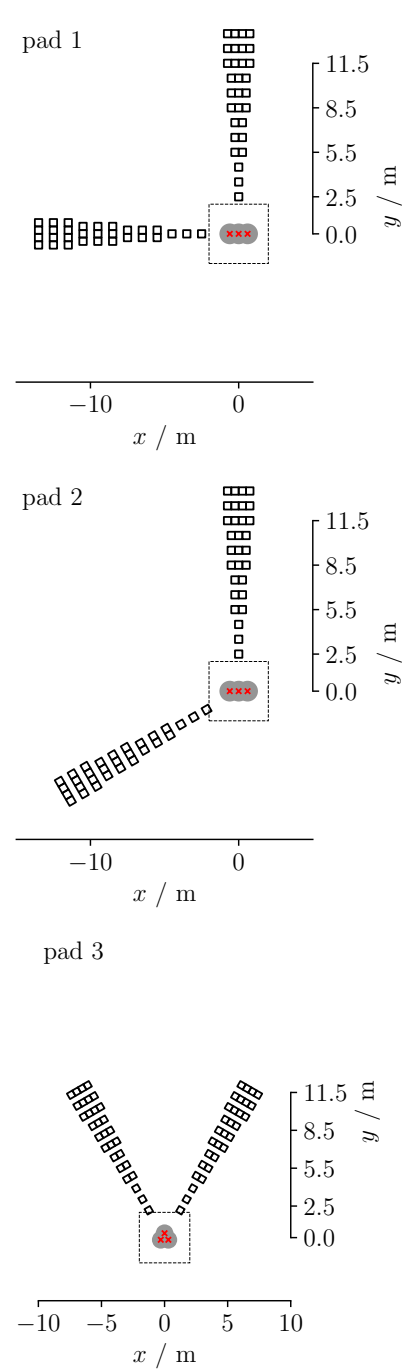

$\operatorname{pad} 4$
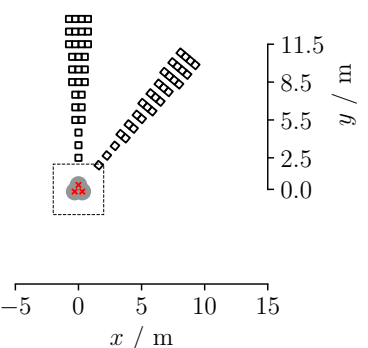
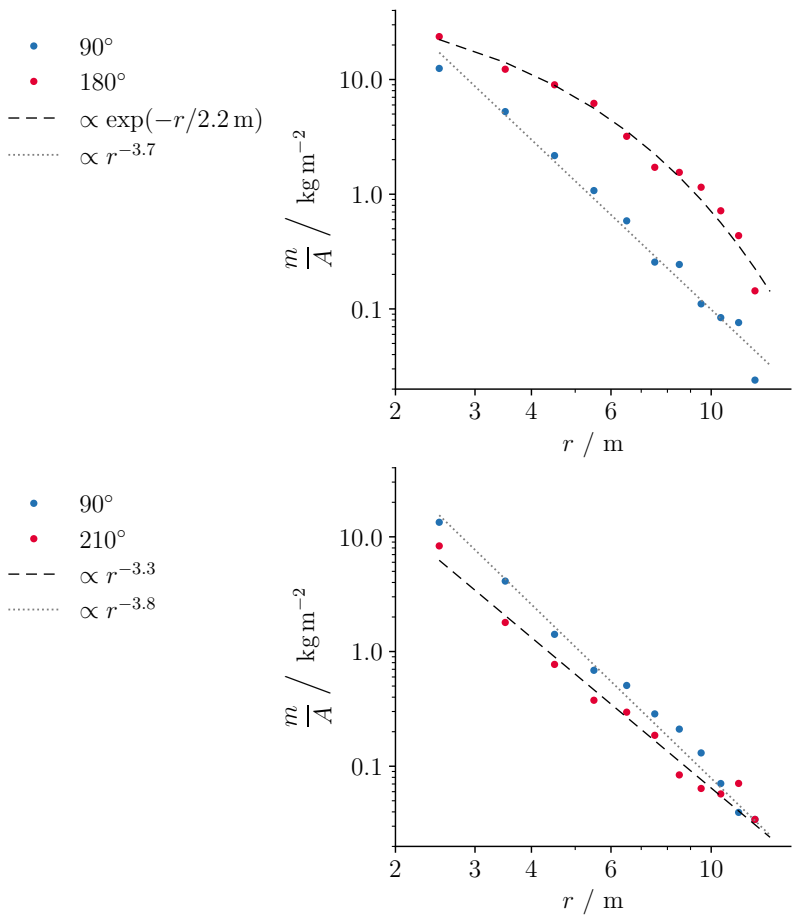

- $60^{\circ}$

- $120^{\circ}$

$---\propto r^{-3.5}$ $\propto r^{-3.1}$

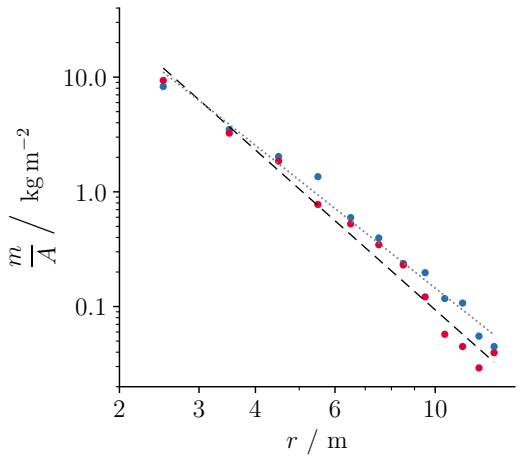

- $90^{\circ}$

- $50^{\circ}$

$---\propto \exp (-r / 3.4 \mathrm{~m})$

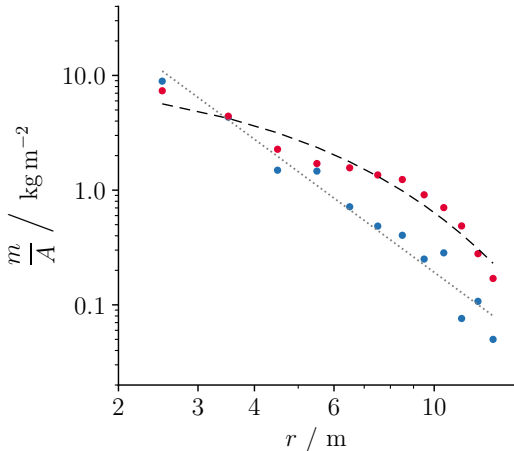

Figure 5: Ejected mass per area at distances $r$ from the crater center for the four blast sequences. Blue points show data of a collection branch in the $\varphi=90^{\circ}$-direction. For pad 1 , the other collection branch was at $\varphi=180^{\circ}$, which was the main ejection direction. This branch follows an exponential decay. The $90^{\circ}$-branch follows a power law in all pads. This branch shows similar decay at higher rates for the linear charge setups in pads 1 and 2 (decays with power $\simeq 3.75 \pm 0.3$ ), and lower decays rates for the triangular charge setups in pads 3 and 4 (decays with power $\simeq 3.0 \pm 0.3$ ). 


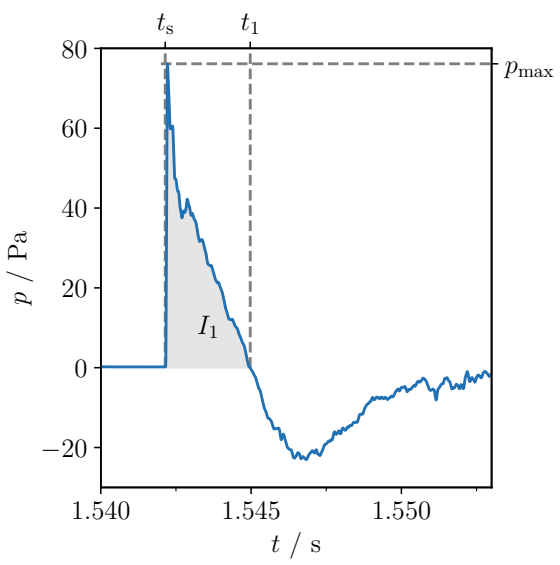

Figure 6: Typical waveform of a blast pulse as recorded by the acoustic microphones; here shown is blast $\# 2$ of pad 2 , at $82 \mathrm{~m}$ distance from source (microphone channel 17). Also shown are characteristic times $t_{\mathrm{s}}$ (shock arrival), $t_{1}$ (first zero crossing), maximum pressure $p_{\max }$ and impulse of the positive pulse part $I_{1}$, that are formulated in Equations 5 and 6 .

\subsection{Seismo-acoustics}

The explosion creates a pressure pulse that propagates faster than- or at the speed of sound. Close to the source the pressure jumps (rises discontinuously) from ambient (atmospheric) value to a maximum and then relaxes back before sinking below ambient pressure (Figure 6) and again relaxing back. At larger distances the propagation speed approaches the speed of sound and the pressure discontinuity relaxes to a steep, but finite slope.

The recorded data show strong air-to-ground and weak ground-to-air wave coupling. A high-frequency signal occurs in the seismic waveforms in close time correlation with the main blast pulses measured in air at the same location by infrasound and sonic range microphones (Figure 7a-d).

\subsubsection{Radial Dependency of Airborne Blast Pulse}

Using features of wave-forms recorded by microphones and/or seismic sensors it is possible to estimate the blast's energy, provided that scaling laws assumed in such models are valid. The scaled peak pressure and scaled impulse of a blast in air depends on the scaled distance where the pressure is measured (Kinney \& Graham, 1985). This relationship can be used to determine the scaled distance of each microphone record, and with that the energy of each blast wave can be estimated. This resource will be used as a reference model, and referred to as KG85 data (or -model). For these blasts in air, the main fundamental three quantities to be scaled are distance, time, and pressure. As in the case for underground blasts distances can be scaled with blast energy $E_{\mathrm{b}}$. Additionally, the relatively high atmospheric homogeneity allow further specification of the atmospheric density, which is often written in terms of transmission factors for scaled distance and time. Scaled distance, time, and pressure are given by

$$
\bar{r}=\frac{f_{d} r}{E^{1 / 3}} \quad, \quad \bar{t}=\frac{f_{t} t}{E^{1 / 3}} \quad, \quad \bar{p}=\frac{p}{p_{\mathrm{a}}},
$$



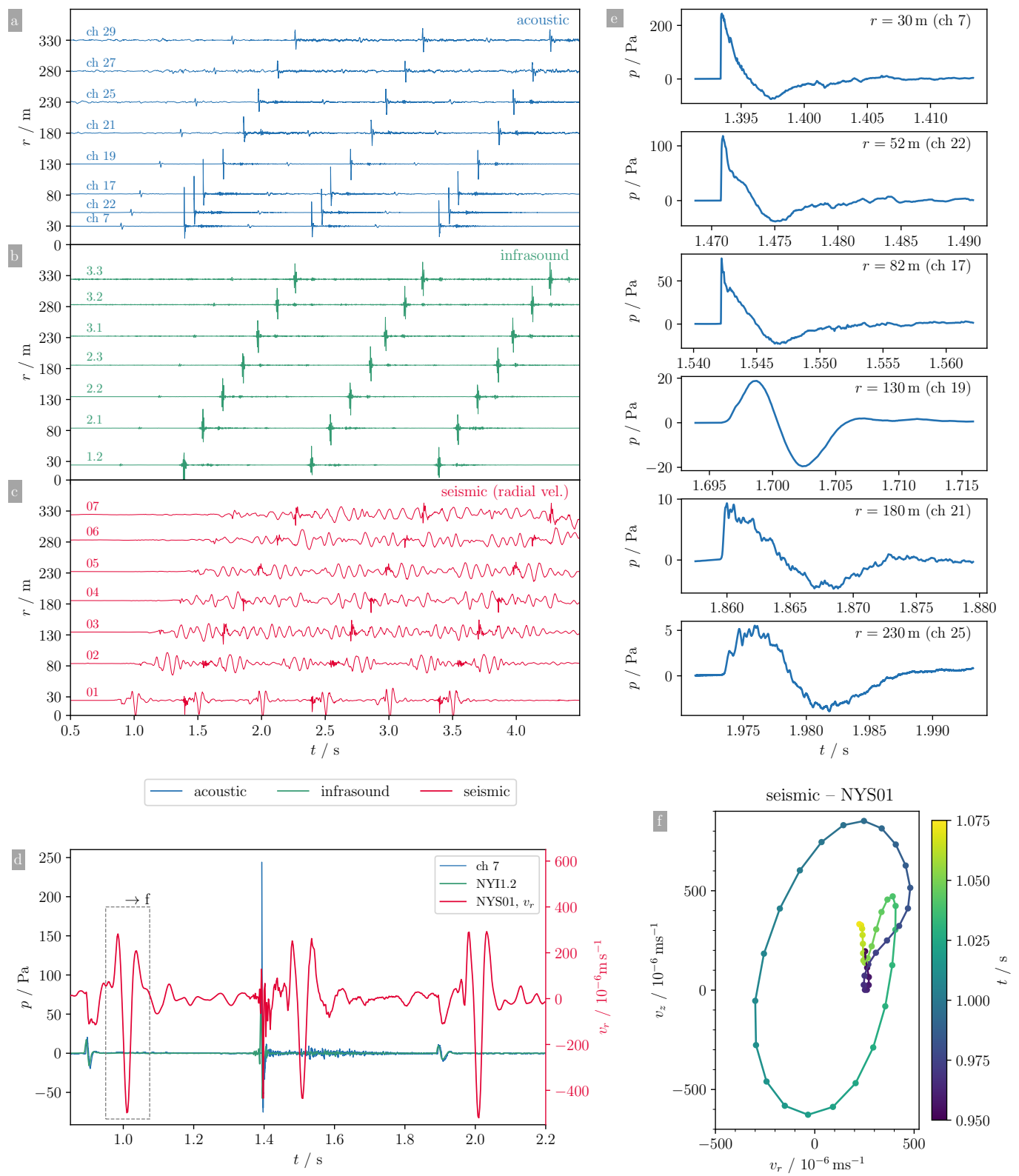

Figure 7: Seismic- infrasound- and acoustic waveform signals of the pad 2 blast sequence. a, b, c: The seismic signals show high-frequency coupling at time and location of the large pressure pulses occurrence at the infrasound- and acoustic microphones. d: First three pulses at horizontal distance $r=30 \mathrm{~m}$. High amplitude air-borne pressure waves, such as the acoustic (blue) and infrasound (green) signals at $t \simeq 1.6 \mathrm{~s}$ correlate better with high frequency signal of the seismic channel compared to lower amplitude pulse signals at about $1.1 \mathrm{~s}$ and $2.2 \mathrm{~s}$. e: Waveforms of microphone records of blast \#2 show a clear transition at distance $<130 \mathrm{~m}$. The $130 \mathrm{~m}$ station recorded a more symmetric signal, while at $180 \mathrm{~m}$ the rising slope was steeper (asymmetric) again. f: Particle motion of the incoming Rayleigh wave created by blast $\# 1$. The time window picked for the radial and vertical components is indicated by the dashed rectangle in $\mathrm{d}$. 
where $p_{\mathrm{a}}$ is the atmospheric pressure and the transmission factors $f_{d}, f_{t}$ for distance and time, respectively, take the density into account in which the blast pulse propagates. They are given by

$$
f_{d}=\left(\frac{\rho}{\rho_{0}}\right)^{1 / 3}=\left(\frac{p_{\mathrm{a}} T_{0}}{p_{0} T}\right)^{1 / 3} \quad, \quad f_{t}=\left(\frac{\rho}{\rho_{0}}\right)^{1 / 3} \frac{c}{c_{0}}=\left(\frac{p_{\mathrm{a}}}{p_{0}}\right)^{1 / 3}\left(\frac{T}{T_{0}}\right)^{1 / 6} .
$$

The index ${ }_{0}$ refers to values of a known blast case. The model only applies to explosive shocks in air. Our recorded pressure pulses show most of the characteristic features of a free air explosion, indicating that enough energy was not contained in the ground, so that an estimate of the un-contained energy, $E_{\mathrm{a}}$, which created a shock pulse in the atmosphere, seems appropriate. Comparison to the known yield of the detonation charges, $E_{\mathrm{b}}$, can then give information of the effect of explosion depth.

Another widely used quantity to measure a blast's intensity, damage potential and energy is its impulse per crossectional area (Schnurr et al., 2020; Guzas \& Earls, 2010; Kinney \& Graham, 1985; Bush et al., 1946), which can be obtained as the time integral of the initial positive pressure peak of a microphone pressure curve as

$$
I_{1}=\int_{t_{\mathrm{s}}}^{t_{1}} p d t
$$

Here $t_{\mathrm{s}}$ is the start time (time of arrival of the pulse at the sensor's location) and $t_{1}$ is the time of first zero crossing of the pressure curve (Figure 6). This time interval always contains the peak pressure. The corresponding scaled impulse is a compound of scaled pressure and time components

$$
\bar{I}_{1}=\int_{\bar{t}_{\mathrm{s}}}^{\bar{t}_{1}} \bar{p} d \bar{t}=\frac{f_{t}}{p_{\mathrm{a}} E_{\mathrm{b}}^{1 / 3}} I_{1}
$$

The KG85 data provides values up to a scaled distance of $3.1 \mathrm{~m} \mathrm{~J}^{-1 / 3}\left(500 \mathrm{~m} \mathrm{~kg}^{-1 / 3}\right)$. According to this dataset the scaled pressure and scaled impulse decay with $1 / \bar{r}$ at relatively large distances $\left(\bar{r} \gtrsim 10^{-2} \mathrm{~m} \mathrm{~J}^{-1 / 3}, 20 \mathrm{~m} \mathrm{~kg}^{-1 / 3}\right)$. The explicit values for the decay are

$$
\begin{array}{cc}
\bar{p}=\frac{a_{p, \text { ref }}}{\bar{r}} \quad, \quad a_{p, \text { ref }}=5.135 \times 10^{-3} \mathrm{~m} \mathrm{~J}^{-1 / 3}, \\
\bar{I}_{1}=\frac{a_{I, \text { ref }}}{\bar{r}} \quad, \quad a_{I, \text { ref }}=5.923 \times 10^{-8} \mathrm{~m} \mathrm{~s} \mathrm{~J}^{-2 / 3} .
\end{array} .
$$

As is common in the analysis of blast waves (Garces, 2018; Kinney \& Graham, 1985), peak pressures were not directly read as the maximum of the measured pressure curve, but impulse $I_{1}$ was calculated and compared to a function representing a blast pulse shape. We used a modified Friedlander shape $\bar{p}(t)=\bar{p}_{\mathrm{p}}\left(1-\frac{t-t_{\mathrm{s}}}{t_{1}-t_{\mathrm{s}}}\right) \exp \left(-\alpha \frac{t-t_{\mathrm{s}}}{t_{1}-t_{\mathrm{s}}}\right)$, see e.g. Marchetti et al. (2013). The value of $p_{\mathrm{p}}$ that fits the measured $I_{1}$ best was used for the peak overpressure. The impulse reference data are somewhat unclear, since the given interpolation function (Appendix B) deviates from the given data points by 17\%. The proportionality constant $a_{I, \text { ref }}$ in Equation 8 is a modified value that takes this into account and is a better fit to the provided reference data.

The more contained blasts did not create large enough blast pulses to make a reasonable comparison with the KG85 reference data. However, all initial and the perceived louder blasts of pads 2 and 4 (blasts 2, 4,6) created wave forms that were consistent with blast pulses and could be compared. In those cases peak pressure data were in agreement with a $1 / r$ dependency at distances of up to $100 \mathrm{~m}$. The impulse data stay consistent up to about $130 \mathrm{~m}$ distance (Figure 9a and b). At larger distances the values deviate significantly from $1 / r$. 


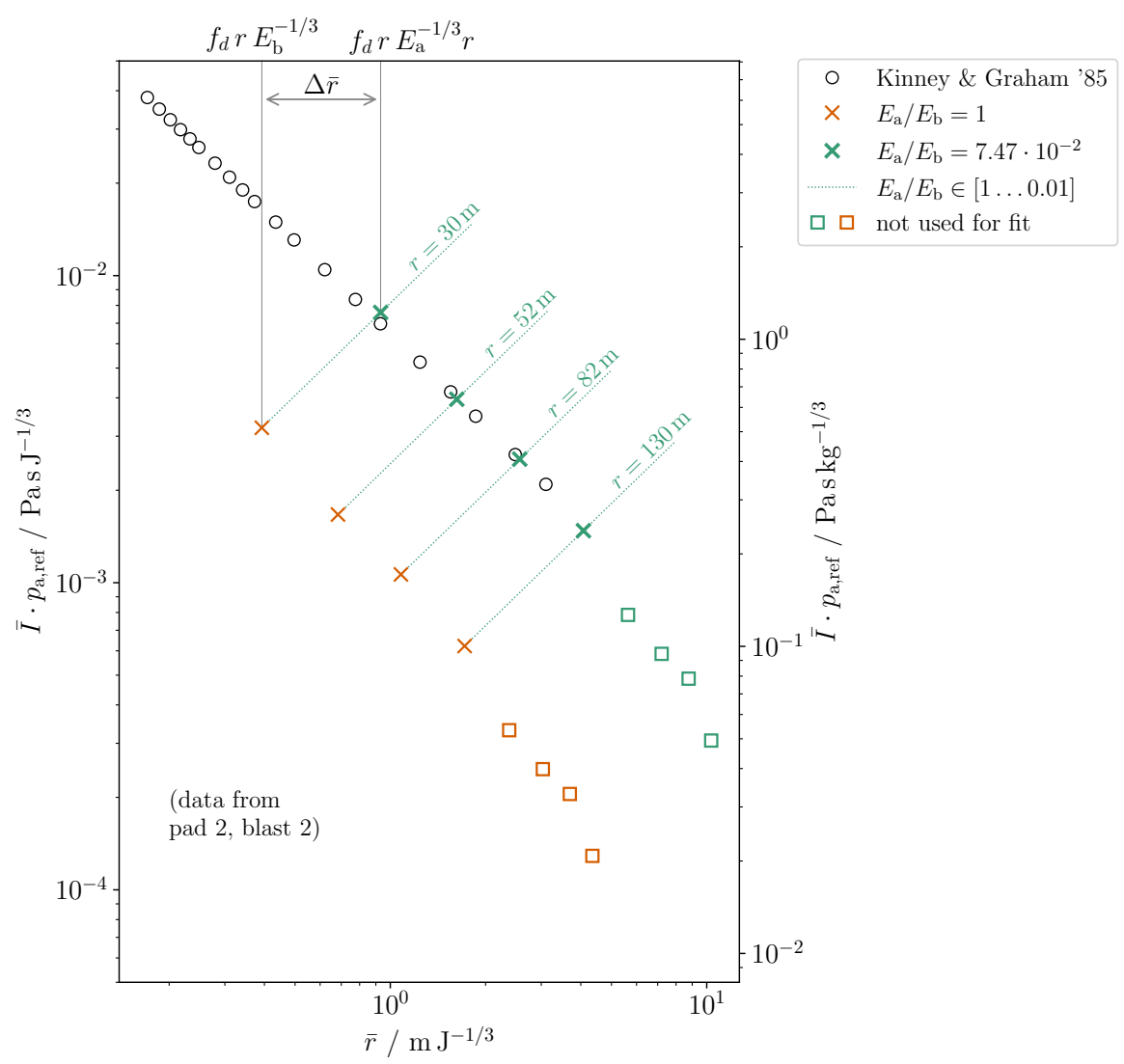

Figure 8: Effect of blast confinement illustrated by a scaled impulse vs. scaled distance plot. Straight forward calculation of scaled distance using the blast's total energy $E_{\mathrm{b}}$ puts the measured scaled impulse (red markers) below the reference values (black circles). The fitting procedure moves the measured values along the green lines. Since both, impulse and distance scale with $E^{-1 / 3}$ their scaled values increase if $E$ decreases. Green markers show values for minimum deviation from reference which correspond to energy $E_{\mathrm{a}}$.

To compare the measured impulse values to the scaled reference, an $r^{-1}$ dependency was fitted to the un-scaled values of a given blast pulse, and the fitting constant $a_{I}$ was used to determine the location in the scaled graph. This determines an energy, $E_{\mathrm{a}}$ ("atmospheric energy"), that creates the pressure pulse:

$$
\begin{aligned}
\bar{I} & =\frac{a_{I, \mathrm{ref}}}{\bar{r}}=\frac{a_{I, \mathrm{ref}} E_{\mathrm{a}}^{1 / 3}}{f_{d} r} \\
& =\frac{f_{t}}{p_{\mathrm{a}} E_{\mathrm{a}}^{1 / 3}} I=\frac{f_{t}}{p_{\mathrm{a}} E_{\mathrm{a}}^{1 / 3}} \frac{a_{I}}{r} \\
E_{\mathrm{a}} & =\left(\frac{f_{d} f_{t}}{p_{\mathrm{a}}} \frac{a_{I}}{a_{I, \mathrm{ref}}}\right)^{3 / 2}
\end{aligned}
$$

Since both, distance and impulse scale with $E_{\mathrm{a}}^{1 / 3}$, the procedure 'moves' values on either axis when changing energy (Figure 8). The result are scaled distances at the end of the KG85 reference scale $\left(\bar{r} \gtrsim 0.6 \mathrm{~m} \mathrm{~J}^{-1 / 3}, 100 \mathrm{~m} \mathrm{~kg}^{-1 / 3}\right)$. From the scaled distance $\bar{r}$ a real distance $r$ corresponds to the energy $E_{\mathrm{a}}=(r / \bar{r})^{3}$, which can be interpreted as the 
energy not contained in the ground, and is smaller compared to the blast energy $E_{\mathrm{b}}$. $E_{\mathrm{a}}$ was found to be around $1.5 \%$ of $E_{\mathrm{b}}$ for the initial blasts, and about $5-7.5 \%$ of $E_{\mathrm{b}}$ for the loud blasts in pads 2 and 4 (Figure $9 \mathrm{c}$ and d, Table 3 ).

Ford et al. (2014) determined distance- and depth dependent energy partitioning of explosions above and below ground using a model for the airborne signal that, after some re-formulation (Appendix C), can be written as

$$
\bar{I}_{1}=\frac{b_{1}}{\bar{r}} \frac{e^{-\bar{d} / \bar{d}_{3}}}{\left(1+e^{-10 \bar{d} / \bar{d}_{3}}\right)^{1 / 10}}
$$

Here $b_{1}=1.15 \times 10^{-7} \mathrm{~s} \mathrm{~m} \mathrm{~J}^{-2 / 3}$ and $\bar{d}_{3}=1.2 \times 10^{-3} \mathrm{~m} \mathrm{~J}^{-1 / 3}$. Evaluated at $\bar{d}=0$ this model expects a ca. $7 \%$ smaller scaled impulse (factor $2^{-1 / 10}, \simeq 0.93$ ) at a given distance compared to a free air blast. A larger discrepancy exists with respect to the KG85 data: The two constants for the $\bar{r}^{-1}$ dependency, $a_{I, \text { ref }}, b_{1}$ differ by a factor 0.51 . Eval-

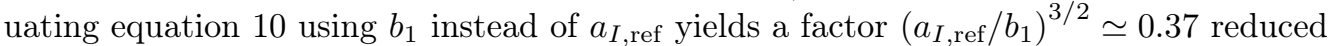
values for $E_{\mathrm{a}}$. The dataset presented here does not contain a zero depth or free air blast, and therefore cannot decide for one of the models. Energy values listed in Table 3 used the KG85 constant, and should be adjusted if used in connection with Equation 11.

\subsubsection{Blast Energy, Charge Depth and Explosion Sequence}

Equation 11 and microphone records of previous blast sessions, carried out in very similar host materials and with similar explosives, show that scaled impulse decays rapidly with scaled depth (Appendix A). A somewhat more accurate match with experimental data is obtained for the peak pressure dependency on depth. Therefore the following is formulated using a peak pressure dependency. At depths $\bar{d}<5 \times 10^{-3} \mathrm{~m} \mathrm{~J}^{-1 / 3}$ peak pressure can be approximated by a product of an exponential which contains the depth part and an amplitude containing the radial dependency:

$$
p_{\mathrm{p}}=A(\bar{r}) e^{-\bar{d} / \bar{d}_{0}}
$$

Here the scaled depth related constant $\bar{d}_{0}=5.4 \times 10^{-4} \mathrm{~m} \mathrm{~J}^{-1 / 3}$. This approximation is valid for scaled depths smaller than $5 \times 10^{-3} \mathrm{~m} \mathrm{~J}^{-1 / 3}$ (Figure A1).

The first charge of a blast sequence detonated under a flat surface in unaltered host material. The following charges detonated under changed topography and somewhat altered host material, since their lateral spacing $\left(0.6 \mathrm{~m}, 8 \times 10^{-3} \mathrm{~m} \mathrm{~J}^{-1 / 3}\right)$ corresponds approximately to the maximum crater radius for that blast energy, and similarly, the vertical spacing $\left(0.3 \mathrm{~m}, 4 \times 10^{-3} \mathrm{~m} \mathrm{~J}^{-1 / 3}\right)$ had, approximately, the optimum depth. Previous experiments showed that for such scaled distances the blast's jet changes shape and, if the topography above the charge has an overall orientation, it will also change direction (Valentine et al., 2015; Ross et al., 2013). If the pre-blast topography is known, parts of the altered surface morphology can be accounted for by the use of an effective scaled depth (Sonder et al., 2015). In case of $0.5 \mathrm{~s}$ blasting delays the topography is however not known. However, the Sonder et al. (2015) analysis also shows that an effective explosion depth rarely deviates by more than $10-20 \%$ from the distance to the closest point to the surface, which is typically the crater bottom. With this approximation, i.e. neglecting the crater shape but not its depth, it is possible to evaluate Equation 12 for peak pressures of blasts that were shot at same lateral location for the two different blast delays, $0.5 \mathrm{~s}$ and $1.5 \mathrm{~s}$ that where realized.

For the pad 1 and 3 experiments this applies to the following pairs of blasts: $(1,4)$, $(2,5)$, and $(3,6)$. For the pad 2 and 4 experiments the blast pairs with same lateral location are $(1,2),(3,4)$ and $(5,6)$. Evaluating Equation 12 for two peak pressures at same scaled distance leaves only the scaled depth to change. For example, considering the ra- 

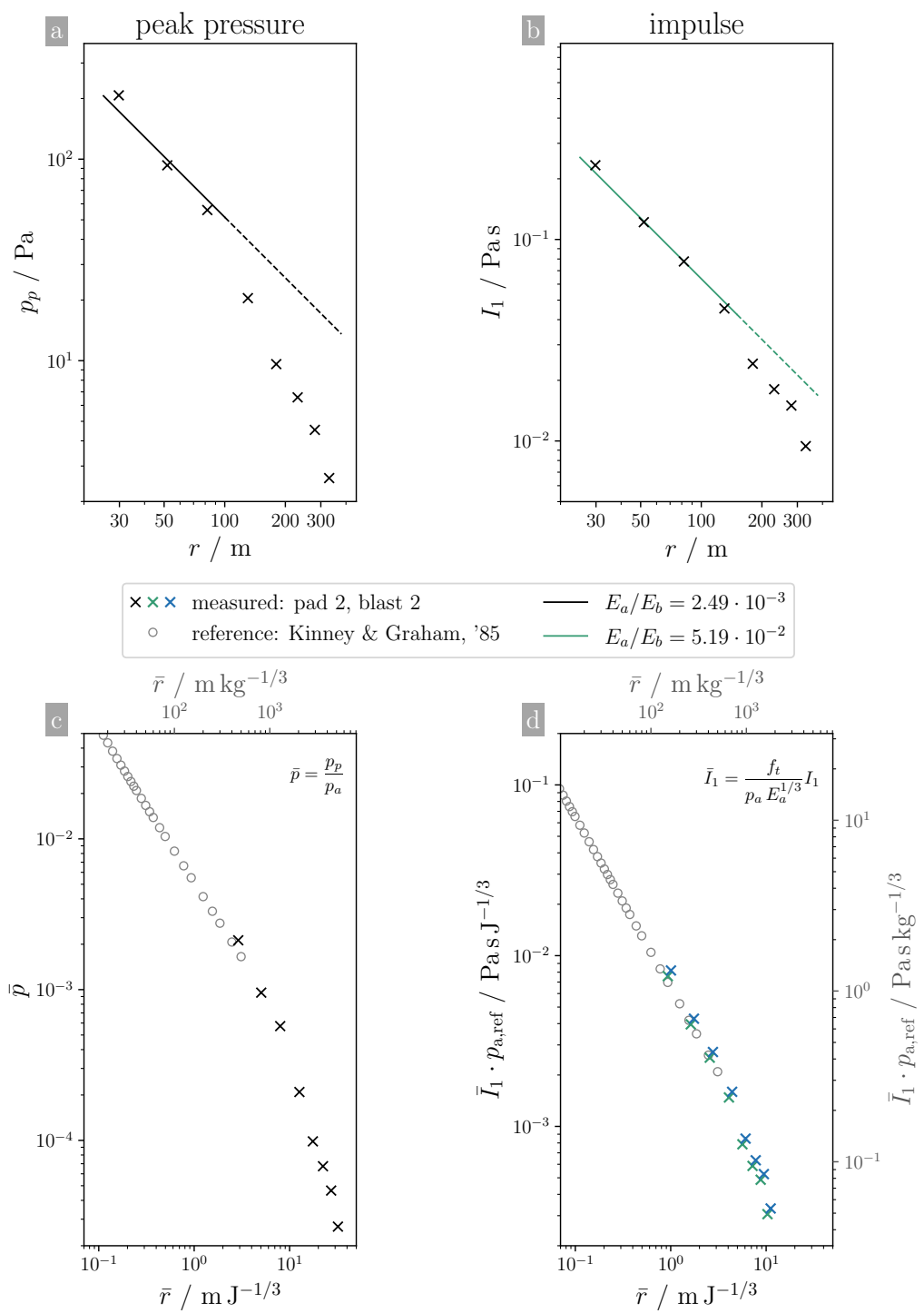

467

468

469

470

471

472

473

474

476

Figure 9: Comparison of peak pressure $p_{\mathrm{p}}$ and impulse $I_{1}$ with respect to their applicability to estimate an explosion energy, and their compatibility to the scaled air blast data by Kinney \& Graham, 1985 (KG85). a, b: The impulse data show a better agreement with the $r^{-1}$-trend. Energies $E_{\mathrm{a}}$ estimated from peak pressures are about a factor 10 smaller compared to the impulse-based estimates. The $p_{\mathrm{p}}$-values start to deviate significantly from the $r^{-1}$-trend at distances $r>100 \mathrm{~m}$. The impulse values start deviating for distances $r>150 \mathrm{~m}$. c: Only the largest blasts produced scaled peak pressures that are comparable to the KG85 values. d: Scaled impulse values show a larger overlap with KG85. This is partially caused by the larger energy estimates, which reduce the scaled impulse and the scaled radius. 
Table 3: Results of the acoustic signal analysis: Acoustic energy, $E_{\mathrm{a}}$, its part of total blast energy, and reduced depths for all experiments. Only signals from the radial microphone line were used. All $E_{\mathrm{a}}$ values were derived from a fit to the impulse-distance relationship (Equations 6 and 8). Only $I_{1}$-values that followed an $r^{-1}$-dependency were used for the fit (Figure 9). For the loud blasts of pads 2 and 4 (blasts 2, 4,6) the $r^{-1}$ dependency ended for $r>130 \mathrm{~m}$. which was the case for microphones at distances up to $130 \mathrm{~m}\left(\bar{r} \leq 1.71 \mathrm{~m} \mathrm{~J}^{-1 / 3}, 276 \mathrm{~m} \mathrm{~kg}^{-1 / 3}\right)$.

\begin{tabular}{|c|c|c|c|c|c|c|c|}
\hline Pad & Blast & $\begin{array}{l}\text { mics } \\
\text { used }^{1}\end{array}$ & $\begin{array}{c}\text { Distance } \\
\text { range }^{2}(\mathrm{~m})\end{array}$ & $\begin{array}{c}E_{\mathrm{a}} \\
\times 10^{3} \mathrm{~J}\end{array}$ & $\begin{array}{c}E_{\mathrm{a}} / E_{\mathbf{b}} \\
\%\end{array}$ & $d_{\text {red }}$ & $\begin{array}{c}\bar{d}_{\mathrm{red}} \\
\times 10^{-3} \mathrm{~m} \mathrm{~J}^{-1 / 3}\end{array}$ \\
\hline \multirow{6}{*}{1} & 1 & 6 & $31.2-280$ & $4.32 \pm 0.52$ & $0.99 \pm 0.12$ & 0.30 & 3.95 \\
\hline & 3 & 6 & $31.2-280$ & $3.88 \pm 0.51$ & $0.89 \pm 0.12$ & 0.30 & 3.95 \\
\hline & 2 & 6 & $31.2-280$ & $4.48 \pm 0.80$ & $1.03 \pm 0.18$ & 0.30 & 3.95 \\
\hline & 4 & 4 & $31.2-280$ & $1.71 \pm 0.11$ & $0.39 \pm 0.02$ & 0.36 & 4.72 \\
\hline & 5 & 3 & $31.2-280$ & $2.59 \pm 0.25$ & $0.59 \pm 0.06$ & 0.32 & 4.21 \\
\hline & 6 & 3 & $31.2-280$ & $1.11 \pm 0.06$ & $0.25 \pm 0.01$ & 0.35 & 4.62 \\
\hline \multirow{6}{*}{2} & 1 & 8 & $29.8-330$ & $7.92 \pm 0.49$ & $1.81 \pm 0.11$ & 0.30 & 3.95 \\
\hline & 2 & 4 & $29.8-130$ & $32.62 \pm 1.61$ & $7.47 \pm 0.37$ & 0.20 & 2.67 \\
\hline & 3 & - & - & - & - & 0.30 & 3.95 \\
\hline & 4 & 4 & $29.8-130$ & $33.37 \pm 0.67$ & $7.64 \pm 0.15$ & 0.17 & 2.30 \\
\hline & 5 & 7 & $29.8-330$ & $3.62 \pm 0.44$ & $0.83 \pm 0.10$ & 0.30 & 3.95 \\
\hline & 6 & 4 & $29.8-130$ & $28.92 \pm 1.62$ & $6.62 \pm 0.37$ & 0.19 & 2.44 \\
\hline \multirow{6}{*}{3} & 1 & 6 & $28.1-280$ & $6.17 \pm 1.39$ & $1.41 \pm 0.32$ & 0.30 & 3.95 \\
\hline & 2 & 6 & $28.1-280$ & $6.28 \pm 0.91$ & $1.44 \pm 0.21$ & 0.30 & 3.95 \\
\hline & 3 & 6 & $28.1-280$ & $16.10 \pm 1.83$ & $3.69 \pm 0.42$ & 0.30 & 3.95 \\
\hline & 4 & 3 & $28.1-80.7$ & $3.13 \pm 0.24$ & $0.72 \pm 0.06$ & 0.33 & 4.33 \\
\hline & 5 & 5 & $28.1-280$ & $6.79 \pm 0.68$ & $1.56 \pm 0.16$ & 0.30 & 4.00 \\
\hline & 6 & 3 & $28.1-80.7$ & $4.41 \pm 0.30$ & $1.01 \pm 0.18$ & 0.07 & 5.05 \\
\hline \multirow{6}{*}{4} & 1 & 4 & $48.6-180$ & $5.82 \pm 0.61$ & $1.33 \pm 0.14$ & 0.30 & 3.95 \\
\hline & 2 & 3 & $48.6-130$ & $23.63 \pm 0.79$ & $5.41 \pm 0.18$ & 0.22 & 2.91 \\
\hline & 3 & 4 & $48.6-180$ & $3.66 \pm 0.37$ & $0.84 \pm 0.08$ & 0.30 & 3.95 \\
\hline & 4 & 3 & $48.6-130$ & $25.21 \pm 0.96$ & $5.78 \pm 0.22$ & 0.22 & 2.93 \\
\hline & 5 & 4 & $48.6-180$ & $6.39 \pm 0.31$ & $1.46 \pm 0.07$ & 0.30 & 3.95 \\
\hline & 6 & 3 & $48.6-130$ & $28.80 \pm 1.10$ & $6.60 \pm 0.25$ & 0.24 & 3.22 \\
\hline
\end{tabular}

1: Number of microphones used to fit the radial dependency to the data.

2: Minimum and maximum distance of the microphones used to determine $E_{\mathrm{a}}$. 
tio of peak pressures of pad 2's blasts 2 and 1 relates the scaled depth of blast 2 to the previous one by

$$
\bar{d}_{2, \mathrm{r}}=\bar{d}_{1}-\bar{d}_{0} \ln \frac{p_{\mathrm{p}, 2}}{p_{\mathrm{p}, 1}} .
$$

This formula can be applied to any of the above listed blast couples with consistent results (Figure 10a), showing that the so-derived depths are reduced by a factor 1.5-3, compared to their initial charge location relative to the surface. Since $E_{\mathrm{b}}$ was the same for all blasts, the lower charge at the moment of its detonation can be estimated to be at a depth $d_{\mathrm{r}}=\bar{d}_{\mathrm{r}} E_{\mathrm{b}}^{1 / 3}$ below the crater bottom at that time. And because the location of the lower charge is known to be $0.6 \mathrm{~m}$ below the original surface, the crater bottom can be estimated at $z_{\text {bottom }}=-0.6 \mathrm{~m}+d_{\mathrm{r}}$ (Figure 10b). The two delay times show that $0.5 \mathrm{~s}$ after detonation the crater bottom is deeper than at $1.5 \mathrm{~s}$. At $1.5 \mathrm{~s}$ the crater bottom is about the same location that would be expected from a blast of energy $E_{\mathrm{b}}$ at optimum depth.

Complete Dataset

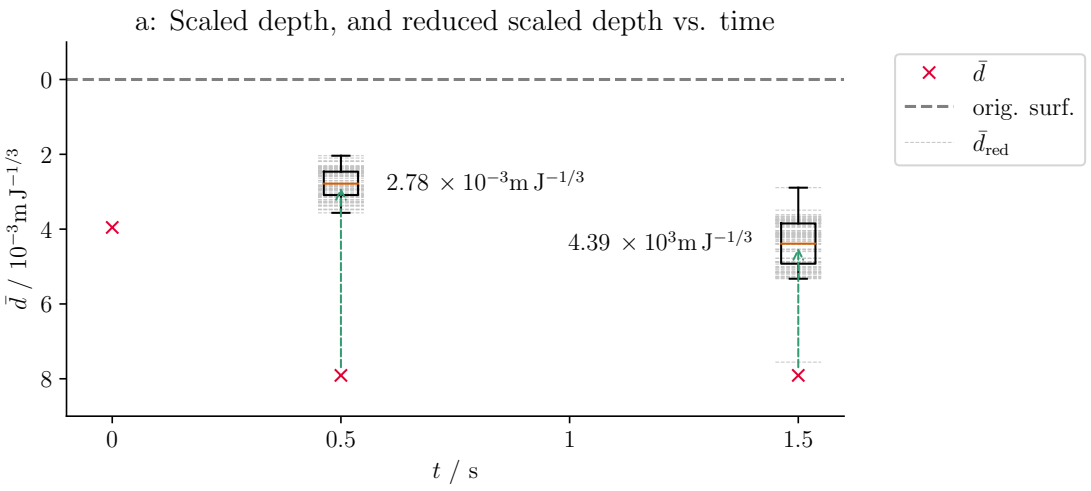

b: Reduced depth based estimate of transient crater bottom

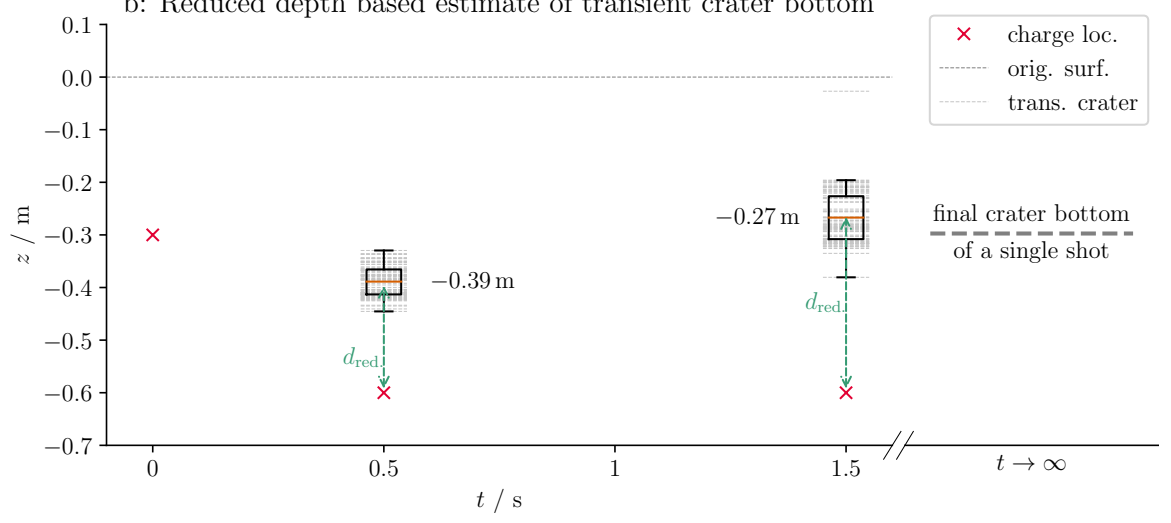

Figure 10: Scaled charge depths (blue crosses), reduced scaled depths (gray, dashed lines: values of a single microphone, orange: average of all microphones) of all microphone sensors at one angle, plotted against time after detonation of the previous charge located vertically above. a: At $0.5 \mathrm{~s}$ delay, scaled depth is reduced by a factor 2-3 compared to original charge location. At $1.5 \mathrm{~s}$ delay scaled depth is only reduced by a factor 1.5-2. (b) Estimated of the time dependent crater bottom evolution. For comparison the dashed gray line shows the measured depth of a single shot of same charge type and energy. 


\begin{tabular}{ccccccccc}
\hline \multirow{2}{*}{ Pad } & \multicolumn{2}{c}{ Measured Footprint } & & \multicolumn{2}{c}{ Reduced Footprint } & & \multicolumn{2}{c}{ Max. Footprint } \\
\cline { 2 - 3 } & $\begin{array}{c}\text { Area } \\
\mathrm{m}^{2}\end{array}$ & $\begin{array}{c}\text { Radius } \\
\mathrm{m}\end{array}$ & & $\begin{array}{c}\text { Area } \\
\mathrm{m}^{2}\end{array}$ & $\begin{array}{c}\text { Radius } \\
\mathrm{m}\end{array}$ & & $\begin{array}{c}\text { Area } \\
\mathrm{m}^{2}\end{array}$ & $\begin{array}{c}\text { Radius } \\
\mathrm{m}\end{array}$ \\
\hline 1 & 3.71 & 0.78 & & 3.97 & 0.81 & & 4.26 & 0.85 \\
2 & 3.38 & 0.73 & & 3.73 & 0.78 & & 4.26 & 0.85 \\
3 & 2.79 & 0.68 & & 3.76 & 0.83 & & 3.92 & 0.85 \\
4 & 3.13 & 0.73 & & 3.71 & 0.82 & & 3.92 & 0.85 \\
\hline
\end{tabular}

\subsubsection{Seismic Signal}

We present here an initial estimate of seismic energy involved in the explosion experiments. A deep analysis of the seismic records will be part of future studies. The energy radiated from a radially symmetric seismic source may be estimated from the measured square velocity of the ground (particle) motion $u_{r}$ (e.g. Boatwright, 1980; Johnson \& Aster, 2005)

$$
E_{\mathrm{s}}=2 \pi r^{2} \frac{\rho_{\mathrm{g}} c_{\mathrm{g}}}{A} \int_{0}^{\infty} S u_{r}^{2}(r, t) d t .
$$

Here $A$ and $S$ are coefficients for signal attenuation and site response, respectively. $\rho_{\mathrm{g}}$ is the ground density and $c_{\mathrm{g}}$ the propagation speed of the ground, both at the observation location. For this first broad look at seismic energy these parameters are assumed to be constant. In this assumed energy estimate only one component of ground motion, radial component $u_{r}$ is non-zero. Other seismic components are therefore ignored in the following. Then $E_{\mathrm{s}}$ can be approximated as

$$
E_{\mathrm{s}} \simeq F r^{2} \int_{0}^{\infty} u_{r}^{2}(r, t) d t \quad, \quad F=2 \pi \rho_{\mathrm{g}} c_{\mathrm{g}} \frac{S}{A}
$$

In this approximation the proportionality factor $F$ depends on a combination of ground properties and attenuation characteristics, but not on $E_{\mathrm{s}}$.

The multi-blast setting adds the difficulty that seismic signals originating from different blasts overlap at larger distances (e.g. for $r \gtrsim 80 \mathrm{~m}$, Figure 7c). From such distances only the cumulative seismic energy of a blast set can be determined:

$$
\sum_{i=0}^{N_{\mathrm{b}}} E_{\mathrm{s}, i}=F r^{2} \int_{0}^{\infty} u_{r}^{2}(r, t) d t \quad\left(\text { here } N_{\mathrm{b}}=6\right) .
$$

At closer ranges the blasts can be identified clearly in the $u^{2}$ signal. There $u^{2}$ decays quickly before the next pulse arrives, and integration over a finite time interval is a valid approximation for each blast (Figure 11a):

$$
E_{\mathrm{s}, i}=F r^{2} \int_{\Delta t_{i}} u_{r}^{2}(r, t) d t
$$



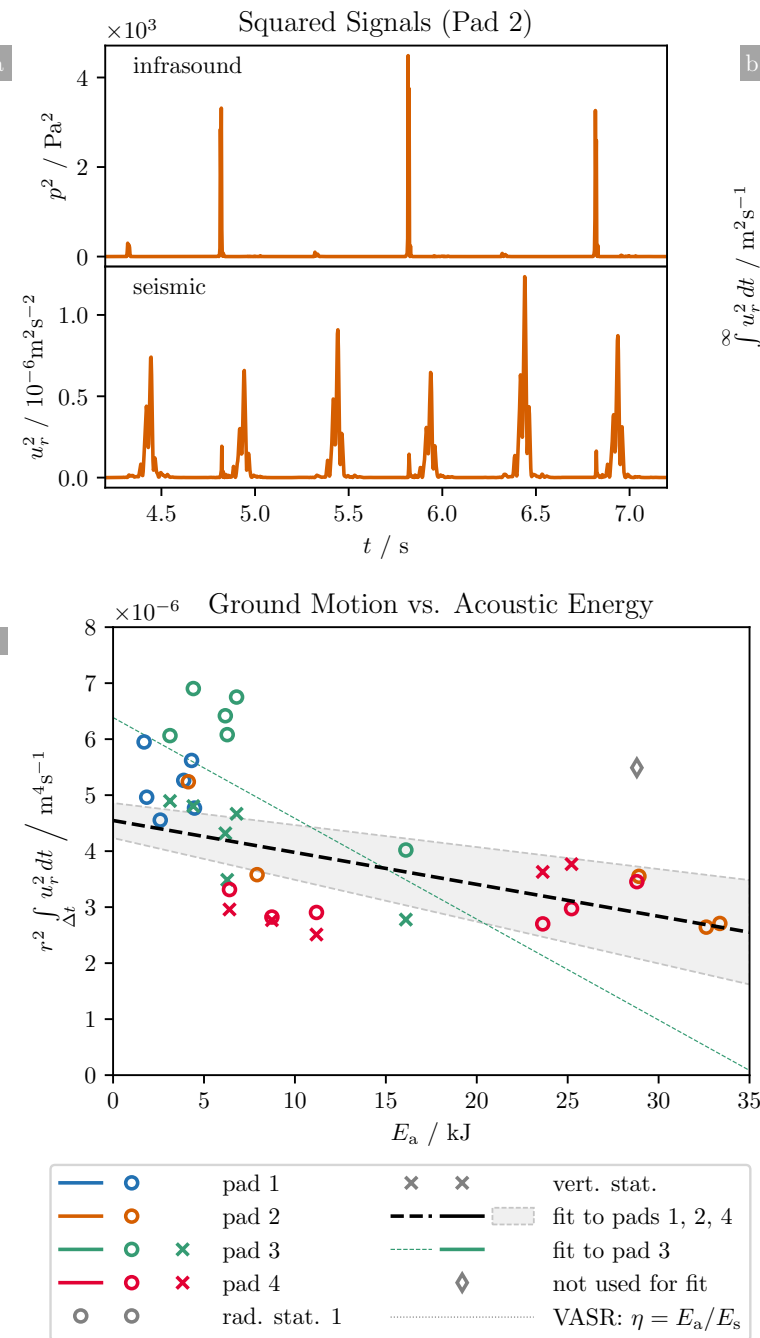

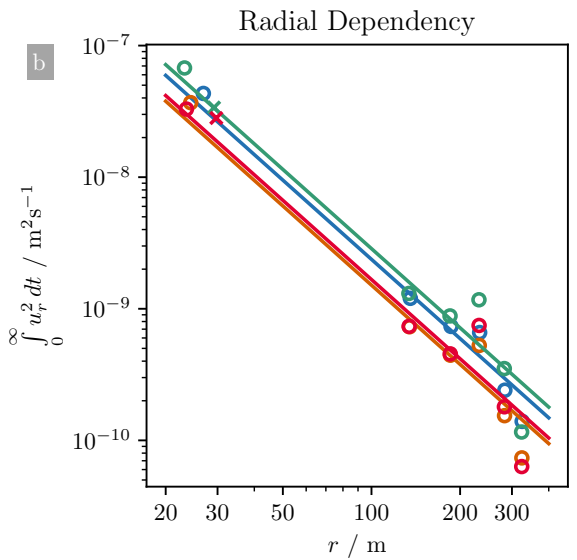

Energy Partitioning

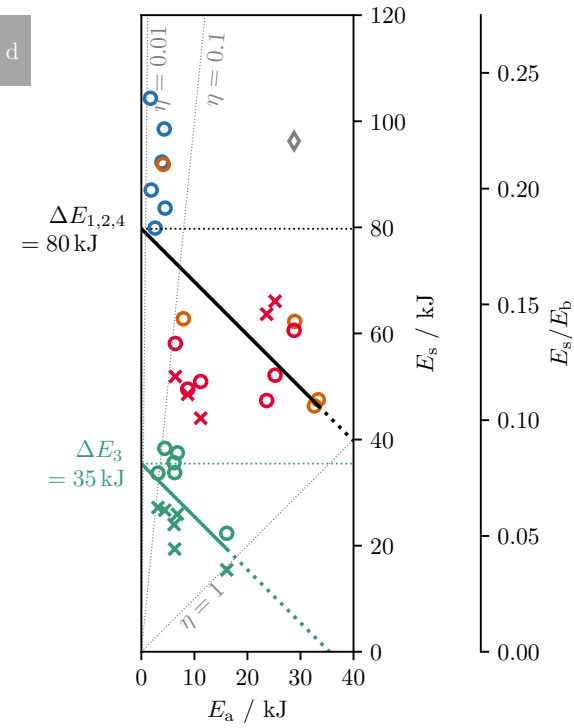

Figure 11: Estimate of seismic energy from squared particle velocity. a: Pad 2 test squared pressure signal of the infrasound sensor and squared particle velocity at first radial station ( $30 \mathrm{~m}$ distance). The seismic signal shows clearly identifiable pulses that can be separated into six time intervals. As described earlier for pad 2 the airborne pressure pulses of blasts 1, 3 and 5 are much weaker as those of blasts 2, 4 and 6 . In contrast peak values of $u^{2}$ are higher for blasts 1, 3 and 5, and somewhat weaker for blasts 2, 4 and 6 . The trend is not as strong for the seismic signal as it is for the airborne signal. b: Radial dependency of squared particle velocity integral. Measured values and fitted $r^{-2}$ curves of Equation 20 are shown. Pads 1 and 3, with sequential shot depth configuration, produced a higher squared particle velocity integral, compared to pads 2 and 4 (interchanging shot depth). To a lesser degree, the triangular pads 3 , and 4 had larger values when compared to the same shot depth configuration of the linear geometrical setups of pads 1 , and 2 . c: Squared particle velocity integral dependency on $E_{\mathrm{a}}$. Despite some scatter, data from pads 1, 2 and 4 follow a common trend, while pad 3 data has a larger slope and offset.

[Caption continues] 
Figure 11: [Continued] The black dashed line is a fit of Equation 19 to data of pads 1, 2 , and 4 . The green dotted line to the pad 3 data. Cross markers show data form radial station $\# 1$, circles data from the vertical station. $\mathbf{d}$ : Seismic energy plotted against acoustic energy for all pads. Black and green lines show the anticipated (linear) relationships using the derived values for $F$ and $\Delta E$. The second vertical axis shows $E_{\mathrm{s}}$ relative to total blast energy $E_{\mathrm{b}}$. The elastic part is ca. $17 \%$ of $E_{\mathrm{b}}$ for pads 1, 2, 4 and ca. $10 \%$ for pad 3. Gray dotted lines show the volcanic acoustic seismic ratio $\eta=E_{\mathrm{a}} / E_{\mathrm{s}}$ (VASR, Johnson \& Aster, 2005). The blasts had VASR values between $10^{-2}$ and 1.

When compared to the airborne signals, the seismic records show an inverted trend: The "muffled" blasts 1, 3 and 5 of pads 2 and 4, which had a much lower airborne signal created a larger seismic signal, when compared to blasts 2, 4 and 6 (Figure reffig:seisa). This behavior serves as motivation for a potential energy partitioning scheme. For a given pad configuration the assumption is made that seismic and acoustic energy of a blast add up to a constant value.

$$
E_{\mathrm{b}}=E_{\mathrm{a}}+E_{\mathrm{s}}+E_{\mathrm{rem}}
$$

In this picture a change in $E_{\mathrm{a}}$ of $\delta E$, for example by a change of blast depth, would result in a change of $E_{\mathrm{s}}$ by $-\delta E$. The remaining energy $E_{\text {rem }}$ stays constant. This energy conservation applies to each blast and to the cumulative case, which allow determination of the two unknowns $F$ and $E_{\text {rem. }}$. With $\Delta E=E_{\mathrm{b}}-E_{\text {rem }}$ the per-blast case becomes

$$
r^{2} \int_{\Delta t_{i}} u_{r}^{2}(r, t) d t=\frac{E_{\mathrm{s}, i}}{F}=\frac{\Delta E-E_{\mathrm{a}, i}}{F},
$$

and the cumulative case is

$$
\begin{aligned}
\int_{0}^{\infty} u_{r}^{2}(r, t) d t & =\frac{N_{\mathrm{b}} E_{\mathrm{b}}-N_{\mathrm{b}} E_{\mathrm{rem}}-\sum E_{\mathrm{a}, i}}{F r^{2}} \\
& =N_{\mathrm{b}} \frac{\Delta E-\left\langle E_{\mathrm{a}}\right\rangle}{F r^{2}},
\end{aligned}
$$

where $\left\langle E_{\mathrm{a}}\right\rangle=\sum E_{\mathrm{a}, i} / N_{\mathrm{b}}$. The difference between the two cases is that for Equation 20 $r$ is treated as independent variable, while in Equation $19 E_{\mathrm{a}}$ is independent. The average value $\left\langle E_{\mathrm{a}}\right\rangle$ is a constant.

The left hand side values of Equation 20 were fitted to an $r^{-2}$ dependency. The result shows the expected behavior: Pads 2 and 4 with the large airborne signals have smaller seismic signals when compared to their respective geometric counterparts pads 1 and 3 (Figure 11b). The per-blast data for the right-hand side of Equation 19 show a different trend of the pad 3 data compared to the other pads (Figure 11c). For small $E_{\mathrm{a}}$ they are larger than the other pads, and then fall off quicker with rising $E_{\mathrm{a}}$. Since for the other pads no unique trend could be determined, pad 3 was treated separately, form pads 1, 2 and 4. For both cases intercept ans slope were determined. Together with the cumulative case fit, values for $\Delta E$ and $F$ were calculated. For pads 1, 2 and $4, \Delta E$ about $17 \%$ of $E_{\mathrm{b}}(\simeq 73 \mathrm{~kJ})$, for pad 3 this value is about $10 \%(\simeq 45 \mathrm{~kJ})$. Highest values of $E_{\mathrm{s}}$ are a factor two larger than highest values of $E_{\mathrm{a}}$. Consequentially in cases of observed higher $E_{\mathrm{a}}$ blasts, seismic and airborne energies were comparable (Figure 11d). To be complete, values for $F$ are $3.5 \times 10^{9} \mathrm{Js} \mathrm{m}^{-4}$ for pads $1,2,4$, and $1.6 \times 10^{9} \mathrm{Js} \mathrm{m}^{-4}$ for pad 3 . 


\section{Discussion}

Any number of subsurface explosions at given lateral location create crater structures ("multiblast craters") of a limited size, determined by the explosion's energy, because any single explosion can eject material only to a finite distance (Sonder et al., 2015). Accordingly, the sizes of the presented craters are larger than one-blast craters, but smaller than they could become when blasting many times at these lateral locations with the same energy. Overlapping footprints from laterally shifting, time separated explosions create compound craters with a footprint area that can be calculated from overlapping circles centered around blast locations (Valentine et al., 2015). For a given explosion depth a radius is related to explosion energy by the scaled radius, and therefore the footprint area is, too. The maximum crater radius that can be realized with many explosions of a given energy is related to the crater radius of one explosion by

$$
r_{\infty, \max }=\frac{r_{1, \max }}{1-e^{-1 / n_{0}}} \simeq 1.49 \bar{r}_{1, \max } E_{\mathrm{b}}^{1 / 3}=0.85 \mathrm{~m},
$$

where $n_{0}=0.9$ is an experimentally determined constant, and $\bar{r}_{1, \max }=7.5 \times 10^{-3} \mathrm{~m} \mathrm{~J}^{-1 / 3}$ is the maximum scaled radius of one explosion, which occurs at the optimum scaled depth (Sonder et al., 2015). The footprint radii measure in this study fit into this picture: they range between $0.68 \mathrm{~m}$ and $0.78 \mathrm{~m}$, which is larger than the single explosion radius $(0.57 \mathrm{~m})$ and smaller than the many-blasts limit. However, the crater sizes are not consistent with respect to the blasting sequence: in case of the linear setup, pad 1 (upper before lower charges) created a larger crater compared to pad 2 (interchanging charge depths), while in case of the triangular setup pad 4 (interchanging depths) created the larger crater when compared to pad 3 (Table 4 ).

Equation 21 can also be used to estimate the final crater size of a hypothetical crater that would be the result of many blasts at reduced depth. It is then necessary to replace the maximum (scaled) crater radius with the reduced radius. The latter can be calculated from the scaled depth dependency, using the scaled reduced depth value. A footprint size estimated this way is larger than the measured two-blast crater, and ca. $7 \%$ smaller compared to the maximum possible crater (Table 4, Figure 12).

Determination of the atmospheric energy $E_{\mathrm{a}}$ from airborne impulse or peak pressure is possible for scaled distances up to about $5 \mathrm{~m} \mathrm{~J}^{-1 / 3}\left(800 \mathrm{~m} \mathrm{~kg}^{-1 / 3}\right)$. At larger distances this type of analysis yields faulty values. A word of caution must be added, since the empirical models by Kinney and Graham (1985) and Ford et al. (2014) yield a factor 2 to 3 different energy estimates. A more in-depth analysis that focuses on the complete seismo-acoustic dataset of the presented experiments may help here. For example, peak pressure of a weak shock (e.g. Young et al., 2015; Muhlestein et al., 2012; Rogers, 1977) decays with a power of radius slightly larger than 1 . Such a dependency may be observed in the presented data (Figure 9a). Other non-linear acoustic factors and nearfield topography may also play a role (Maher et al., 2020). Nevertheless, both models evaluated here result in single digit values for the percentage of the energy ratio $E_{\mathrm{a}} / E_{\mathrm{b}}$. The relatively small amounts of explosives used, have the advantage that analysis does not have to deal with complications arising from drastically changing transmission factors (Equation 4), as in the case of large scale explosive events (e.g. Kim \& Rodgers, 2016, 2017) or volcanic eruptions (Matoza et al., 2009).

The changes in the apparent ("reduced") crater depth over time show that $0.5 \mathrm{~s}$ after detonation the crater is about a factor 1.5 deeper compared to $1.5 \mathrm{~s}$ after detonation. It is not clear whether this is the time of the transient cavity's maximum opening or not. The depth at $1.5 \mathrm{~s}$ is comparable to the depth of a single blast crater. For volcanic activity the timescale on which a crater forms is important. In this period part of the overlying mass confining magma in the ground is reduced, creating an effectively reduced load, changing- or enabling non-steady state processes, such as magma-water mixing and phreatomagmatism (Büttner \& Zimanowski, 1998; Lorenz, 1975) or decompression driven ac- 

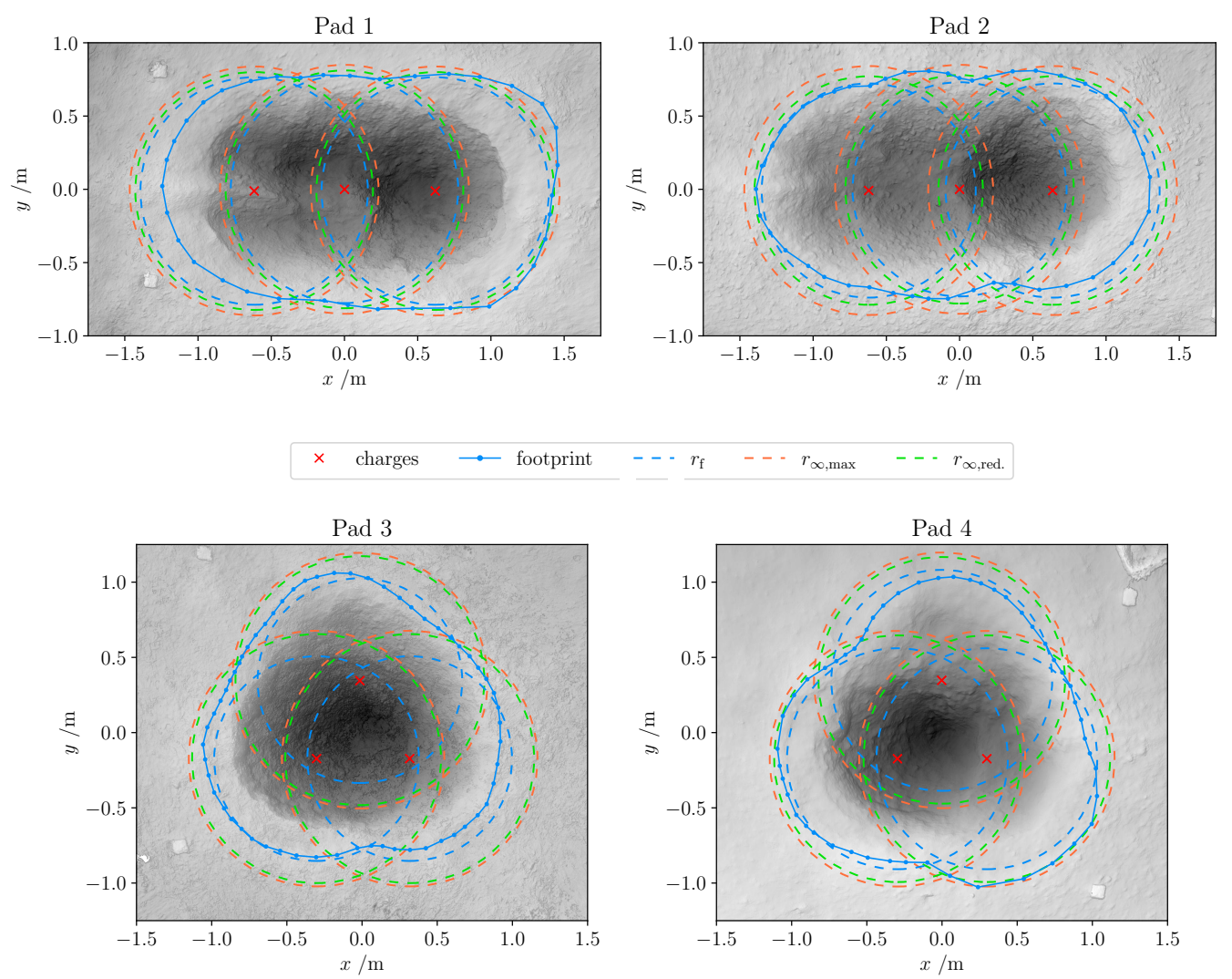

Figure 12: Map views of the four craters, their footprints, and footprint equivalent circles of corresponding radii. All radii correspond to an explosion energy, $E_{\mathrm{b}}=4.635 \times 10^{5} \mathrm{~J}$. Blue lines represent the measured footprint (topographic high) Blue dashed circles are the equivalent radii. Green lines represent the maximum possible footprint that can be expected from this blast energy. Red lines show the hypothetical footprint that would be the result of many explosions at the average reduced depth as measured in each pad. 
tivity (Gonnermann \& Manga, 2007). Assuming for a moment without proof that crater formation duration scales, analog to other blast related time and length (e.g. blast depth, crater radius), with $E_{\mathrm{b}}^{1 / 3}$, the presented results mean that for $E_{\mathrm{b}}=0.4365 \mathrm{MJ}$ crater formation lasts on the order of $1 \mathrm{~s}$, which corresponds to a scaled duration of $1.3 \times 10^{-2} \mathrm{~s} \mathrm{~J}^{-1 / 3}$. An event creating a crater of about $15 \mathrm{~m}$ diameter would need $10^{9} \mathrm{~J}$ (Valentine et al., 2014 ) if created by a single blast, and would be formed in $1.3 \times 10^{-2} \mathrm{~s} \mathrm{~J}^{-1 / 3} \times 10^{3} \mathrm{~J}^{1 / 3}=$ $13 \mathrm{~s}$. A $25 \mathrm{~m}$ diameter crater would then need $44 \mathrm{~s}$ to form. It is, however, likely that other factors complicate such a straight forward scaling approach.

Despite such scaling difficulties the experiments show that explosions which occur at depths previously thought to be contained in the subsurface (Valentine et al., 2014) have to be considered potentially hazardous, if there is a realistic probability that it could occur as a result of crater formation above. The scenario of successively crater deepening, which is also of military interest (Antoun et al., 2003), cannot repeat indefinitely, since the following crater needs to move material from greater depth to the surface in a finite time window, which needs energy. More experiments are necessary to test where this limit lies, and what the exact crater formation duration is.

Analysis of the seismic signal reveals why the pad 3 crater is smaller compared to pad 4: Pad 3 had different attenuation- and coupling conditions leading to less energy available for seismic and acoustic pressure or momentum generation $(\Delta E)$, and more energy dissipated without momentum generation. The different coupling is likely the result of a variation in the pads host properties: On a subjective level, personnel preparing the pad for charge placement before blasting, can confirm that pad 3 'felt' somewhat different compared to the others when punching holes for charge placement into the material. Such unintentional host variability highlights the sensitivity of the crater formation process to host properties (see also Macorps et al., 2016). The estimate of seismic energy and the energy partitioning analysis rely on good knowledge of $E_{\mathrm{a}}$. The assumptions made to estimate $E_{\mathrm{s}}$ work well for large values of $E_{\mathrm{a}}$. At smaller $E_{\mathrm{a}}$ (more contained blasts) scatter becomes larger, which suggests that the underlying assumption, that energy is partitioned only between seismic and airborne signal producing effects, does not apply there. The squared velocity- and pressure signals of pads 1 and 3 emphasize this trend (Supporting Information Figure S9 and Figure 11a). In a first order estimate combination of the available data from the blasts in pads 1, 2 and 4 was between $10 \%$ and $20 \%$ of $E_{\mathrm{b}}$, and between $5 \%$ and $10 \%$ for the blasts of pad 3 . The experiments show how explosive energy is contained by friction, strength and inertia of the surrounding (overlying) material, and how energy translates from driving ground-bound (seismic) to airborne processes, once the overarching containment parameter, scaled depth $\bar{d}$, changes.

\section{Conclusions}

Rapidly-timed subsurface blasts, occur in fields such as mining, geotechnical, military and medical applications (Qiu et al., 2018; Arora et al., 2017; Zhou et al., 2016; Mammadova et al., 2017). Our analysis of the ejecta, crater morphology, and seismo-acoustic signals should be applicable to those situations. We highlight volcanic eruptions, which often involve explosions in rapid succession (Dürig, Gudmundsson, Karmann, et al., 2015; Pistolesi et al., 2011). The results of this study provide insight on how to quantitatively interpret geophysical signals measured during such eruptions, as well as the resulting craters and deposits. They show that energy is a robust parameter to relate the transient, dynamic phenomena, such as airborne and seismic pressure and stress waves and debris jets, with the long term products such as crater, subsurface deposits and ejecta. Finally, we emphasize that much of the presented physical signal analysis relies on (a) the high frequency records of airborne signal and (b) on the combination of relative near-field and far-field records. Deployment of such sensors hold promise for progress in seismo-acoustic volcano monitoring. 


\section{Appendix A Depth- and Distance Dependency of Peak Pressures from Previous Experiments}

In previous blasting experiments (Ross et al., 2013; Graettinger et al., 2014; Valentine et al., 2015; Sonder et al., 2015), a set of uncalibrated microphones was placed every $5 \mathrm{~m}$ starting at $5 \mathrm{~m}$ to $30 \mathrm{~m}$ distance from the source. In all experiments the microphones were placed $10 \mathrm{~cm}$ above the ground facing towards the blast center. The blasts happened at various scaled depths with an emphasis roughly around optimum excavation conditions $\left(\bar{d} \simeq 4 \times 10^{-3} \mathrm{~m} \mathrm{~J}^{-1 / 3}\right)$, but also deeper and some shallower blasts. Despite the uncalibrated pressure signal the raw signals were evaluated, since all sensors were of same model and therefore comparable. The result can be used to determine the relative depth dependency of impulse- and pressure signals, and compare them to other work (e.g. Ford et al., 2014). Signals were evaluated for peak pressure and impulse the same way as described for the here presented experiments in the main text.

Results show that the expected exponential depth dependency (Equation 11) underestimates both, pressure and impulse for deeper blasts (Figure A1). Therefore a second term that only depends on scaled distance was added to the combined depth- and distance dependencies

$$
\begin{aligned}
p_{\mathrm{p}}(\bar{d}, \bar{r}) & =\frac{C_{\mathrm{p}, 1}}{\bar{r}} e^{-\bar{d} / \bar{d}_{p, 0}}+\frac{C_{\mathrm{p}, 2}}{\bar{r}}, \\
\bar{I}(\bar{d}, \bar{r}) & =\frac{C_{\mathrm{i}, 1}}{\bar{r}} e^{-\bar{d} / \bar{d}_{i, 0}}+\frac{C_{\mathrm{i}, 2}}{\bar{r}} .
\end{aligned}
$$

At scaled depths smaller than $1.2 \bar{d}_{\text {opt }}\left(\simeq 5 \times 10^{-3} \mathrm{~m} \mathrm{~J}^{-1 / 3}\right)$ the first term dominates, and the peak pressure show an exponential dependency (Figure A1). At larger scaled depths peak pressures decay slower than this exponential predicts. More research is necessary, to clarify the slow decay. Bowman et al. (2014) suggest that ground motion dominates the airborne signal at larger depths. Best fitting values for the depth decay constant in the exponential is for the pressure case $\bar{d}_{p, 0}=(5.4 \pm 0.5) \times 10^{-4} \mathrm{~m} \mathrm{~J}^{-1 / 3}$, and for the impulse case $\bar{d}_{i, 0}=(1.1 \pm 0.3) \times 10^{-3} \mathrm{~m} \mathrm{~J}^{-1 / 3} \cdot \bar{d}_{i, 0}$ deviates by about $12 \%$ from the value found by Ford et al. (2014) responsible for depth decay $\left(\bar{d}_{3}\right.$, Table C1). We interpret this as good agreement for the range $0 \leq \bar{d} \leq 5 \times 10^{-3} \mathrm{~m} \mathrm{~J}^{-1 / 3}$.

\section{Appendix B Interpolation Constants of KG85 Pressure and Impulse}

The empirical equations for dependencies of blast overpressure, scaled impulse and scaled blast duration on scaled distance are as follows.

Overpressure:

$$
\bar{p}=\bar{p}_{0} \frac{1+\left(\frac{\bar{r}}{Z_{p, 0}}\right)^{2}}{\sqrt{1+\left(\frac{\bar{r}}{Z_{p, 1}}\right)^{2}} \sqrt{1+\left(\frac{\bar{r}}{Z_{p, 2}}\right)^{2}} \sqrt{1+\left(\frac{\bar{r}}{Z_{p, 3}}\right)^{2}}}
$$

Scaled impulse:

$$
\bar{I}_{1}=\bar{I}_{0} \frac{\sqrt{1+\left(\frac{\bar{r}}{Z_{I, 0}}\right)^{4}}}{\left(\frac{\bar{r}}{Z_{I, 1}}\right)^{2}\left(1+\left(\frac{\bar{r}}{Z_{I, 2}}\right)^{3}\right)^{1 / 3}}
$$



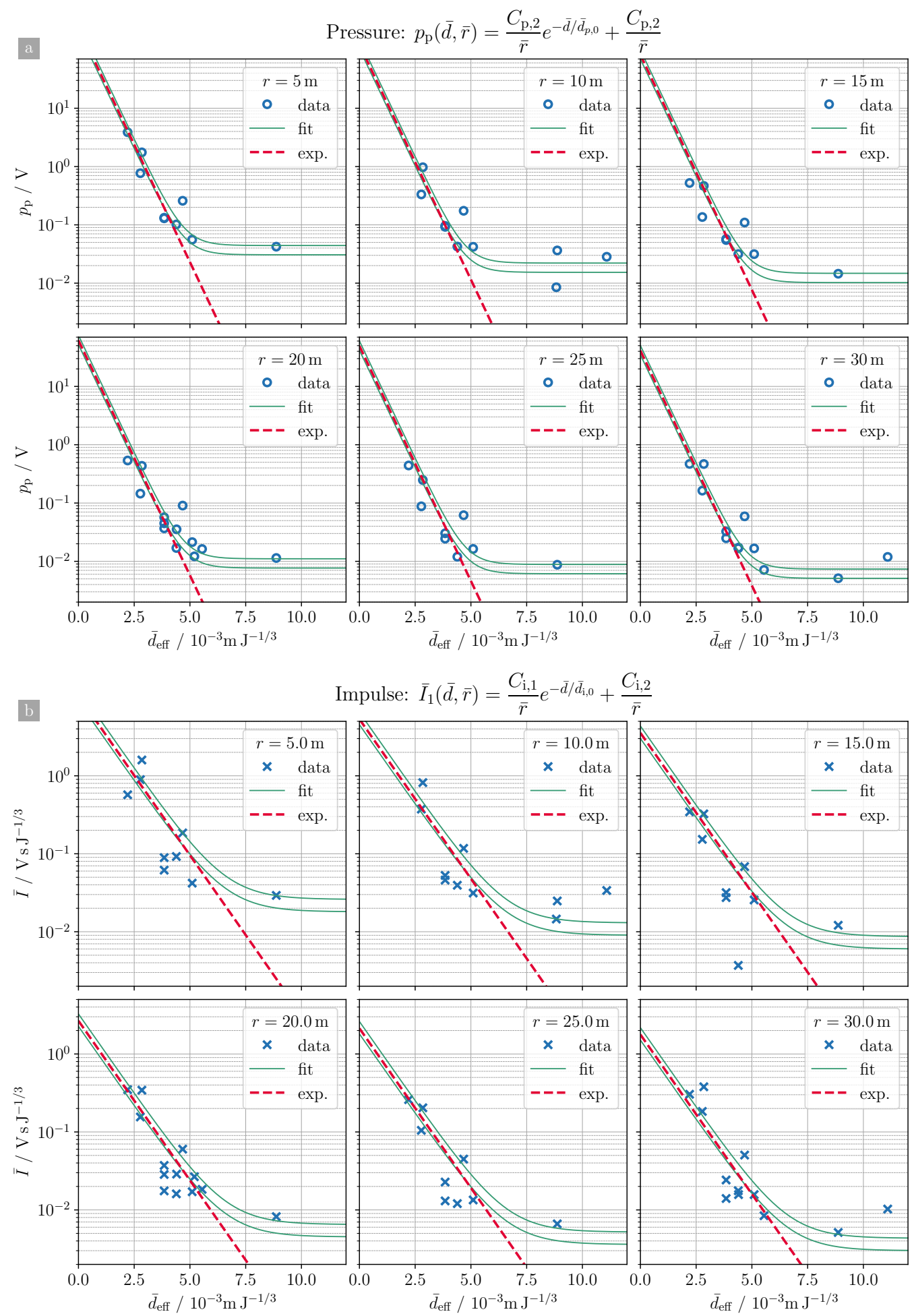

Figure A1: Peak pressure (a) and scaled impulse (b) of previous blast experiments, measured between $5 \mathrm{~m}$ and $30 \mathrm{~m}$ from the blasts. Pressures are shown in raw units (Volts). Depth dependencies are exponential for $\bar{d} \leq 5 \times 10^{-3} \mathrm{~m} \mathrm{~J}^{-1 / 3}$.

[Caption continues...] 
Figure A1: [Continued $]$ Peak pressure decays roughly double as fast compared to impulse $\left(\bar{d}_{p, 0}=5.4 \times 10^{-4} \mathrm{~m} \mathrm{~J}^{-1 / 3}, \bar{d}_{i, 0}=11 \times 10^{-4} \mathrm{~m} \mathrm{~J}^{-1 / 3}\right)$. The red dashed lines are the exponentials $C_{\mathrm{p}, 1} e^{-\bar{d} / \bar{d}_{p, 0} / \bar{r}}$ and $C_{\mathrm{i}, 1} e^{-\bar{d} / \bar{d}_{p, 0}} / \bar{r}$, for pressure and impulse, respectively.

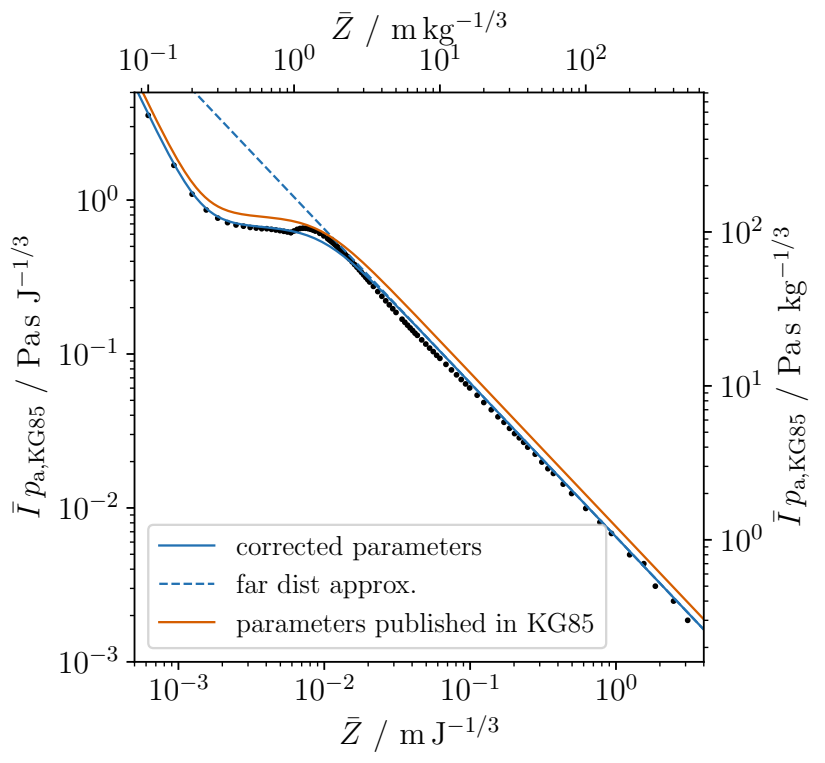

Figure B1: Effect of corrected value for $\bar{I}_{0}$ on the interpolation curve (Equation B2). For a reason not known to the authors the original value for $\bar{I}_{0}$ (orange curve) does not fit the KG85 data (black dots) well. We used a changed value, which better fits this data (blue curves, Table B1).

Scaled blast duration:

$$
\bar{t}_{\mathrm{d}}=\bar{t}_{0} \frac{1+\left(\frac{\bar{r}}{Z_{t, 0}}\right)^{10}}{\left(1+\left(\frac{\bar{r}}{Z_{t, 1}}\right)^{3}\right)\left(1+\left(\frac{\bar{r}}{Z_{t, 2}}\right)^{6}\right)\left(1+\left(\frac{\bar{r}}{Z_{t, 3}}\right)^{2}\right)^{1 / 2}}
$$

Values for the constants $Z_{x, y}$ are given in Table B1. For large distances, i.e. $\bar{r} \gg Z_{x, y}$ the 1 in each of the factors in the above formulas becomes small when compared to the factor $\bar{r} / Z_{x, y}$ and can be neglected. Then $\bar{p}$ and $\bar{I}$ go with $\bar{r}^{-1}$ :

$$
\begin{array}{ll}
\bar{p} \sim \bar{p}_{0} \frac{Z_{p, 1} Z_{p, 2} Z_{p, 3}}{Z_{p, 0}} \frac{1}{\bar{r}}=\frac{a_{p, \text { ref }}}{\bar{r}} \\
\bar{I} \sim \bar{I}_{0} \frac{Z_{I, 1}^{2} Z_{I, 2}}{Z_{I, 0}^{2}} \frac{1}{\bar{r}} \quad=\frac{a_{I, \text { ref }}}{\bar{r}}
\end{array}
$$


Table B1: Constants for the empirical interpolation formulas for blast pulse overpressure, scaled impulse and scaled duration, in SI and kg-TNT equivalent units.

\begin{tabular}{|c|c|c|c|}
\hline \multicolumn{2}{|c|}{ Constant SI } & \multirow{2}{*}{$\begin{array}{ll}\mathrm{kg}_{\text {TNT }} & \\
& \underline{\text { Pressure }}\end{array}$} & \multirow{2}{*}{ Remarks } \\
\hline & & & \\
\hline $\bar{p}_{0}$ & $8.08 \times 10^{2}$ & $8.08 \times 10^{2}$ & \multirow{5}{*}{$\begin{array}{l}\text { Scaled length- and time units differ by a } \\
\text { factor of the } 1 / 3 \text { power of } 1 \mathrm{~kg} \text { TNT } \\
\text { explosive energy. }\left(E_{\mathrm{kg} \mathrm{TNT}}\right)^{1 / 3} \\
=\left(4.184 \times 10^{6} \mathrm{~J}\right)^{1 / 3}=161.1 \mathrm{~J}^{1 / 3} .\end{array}$} \\
\hline$Z_{p, 0}$ & $2.79 \times 10^{-2} \mathrm{~m} \mathrm{~J}^{-1 / 3}$ & $4.50 \mathrm{~m} \mathrm{~kg}^{-1 / 3}$ & \\
\hline$Z_{p, 1}$ & $2.98 \times 10^{-4} \mathrm{~m} \mathrm{~J}^{-1 / 3}$ & $4.80 \mathrm{~m} \mathrm{~kg}^{-1 / 3}$ & \\
\hline$Z_{p, 2}$ & $1.99 \times 10^{-3} \mathrm{~m} \mathrm{~J}^{-1 / 3}$ & $0.32 \mathrm{~m} \mathrm{~kg}^{-1 / 3}$ & \\
\hline$Z_{p, 3}$ & $8.38 \times 10^{-3} \mathrm{~m} \mathrm{~J}^{-1 / 3}$ & $1.35 \mathrm{~m} \mathrm{~kg}^{-1 / 3}$ & \\
\hline \multicolumn{4}{|c|}{ Impulse } \\
\hline $\bar{I}_{0}$ & $3.52 \times 10^{-7} \mathrm{~s} \mathrm{~J}^{-1 / 3}$ & $5.68 \times 10^{-5 \mathrm{~s} \mathrm{~kg}^{-1 / 3}}$ & \multirow{4}{*}{$\begin{array}{l}\text { Original value for } \bar{I}_{0} \text { from Kinney and } \\
\text { Graham }(1985) \text { is } 6.61 \times 10^{-5} \mathrm{~s} \mathrm{~kg}^{-1 / 3}= \\
6.7 \times 10^{-2} \text { bar } \mathrm{ms} \mathrm{kg}^{-1 / 3} / 1.01325 \text { bar. }\end{array}$} \\
\hline$Z_{I, 0}$ & $1.43 \times 10^{-3} \mathrm{~m} \mathrm{~J}^{-1 / 3}$ & $0.23 \mathrm{~m} \mathrm{~kg}^{-1 / 3}$ & \\
\hline$Z_{I, 1}$ & $6.21 \times 10^{-3} \mathrm{~m} \mathrm{~J}^{-1 / 3}$ & $1.00 \mathrm{~m} \mathrm{~kg}^{-1 / 3}$ & \\
\hline$Z_{I, 2}$ & $9.62 \times 10^{-3} \mathrm{~m} \mathrm{~J}^{-1 / 3}$ & $1.55 \mathrm{~m} \mathrm{~kg}^{-1 / 3}$ & \\
\hline \multicolumn{4}{|c|}{ Duration } \\
\hline $\bar{t}_{0}$ & $6.08 \times 10^{-3} \mathrm{~s} \mathrm{~J}^{-1 / 3}$ & $0.980 \mathrm{~s} \mathrm{~kg}^{-1 / 3}$ & \\
\hline$Z_{t, 0}$ & $3.35 \times 10^{-3} \mathrm{~m} \mathrm{~J}^{-1 / 3}$ & $0.54 \mathrm{~m} \mathrm{~kg}^{-1 / 3}$ & \\
\hline$Z_{t, 1}$ & $1.24 \times 10^{-3} \mathrm{~m} \mathrm{~J}^{-1 / 3}$ & $0.02 \mathrm{~m} \mathrm{~kg}^{-1 / 3}$ & \\
\hline$Z_{t, 2}$ & $4.59 \times 10^{-3} \mathrm{~m} \mathrm{~J}^{-1 / 3}$ & $0.74 \mathrm{~m} \mathrm{~kg}^{-1 / 3}$ & \\
\hline$Z_{t, 3}$ & $4.28 \times 10^{-2} \mathrm{~m} \mathrm{~J}^{-1 / 3}$ & $6.90 \mathrm{~m} \mathrm{~kg}^{-1 / 3}$ & \\
\hline
\end{tabular}


Table C1: Constants for the empirical impulse scaling formula from Ford et al., 2014.

\begin{tabular}{|c|c|c|c|c|}
\hline Constant & & & SI & $\mathrm{kg}_{\mathrm{TNT}}$ \\
\hline$\beta_{1}$ & $h_{1}=1$ Pasm & $10^{\beta_{1}}$ & 2.48 & 2.48 \\
\hline$b_{1}$ & $\delta_{1}=\overline{p_{\mathrm{a}, \mathrm{ref}}}$ & $\overline{E_{\mathrm{kg}, \mathrm{TNT}}^{2 / 3}}$ & $1.15 \times 10^{-7} \mathrm{~s} \mathrm{~m} \mathrm{~J}^{-2 / 3}$ & $1.85 \times 10^{-5} \mathrm{~s} \mathrm{~m} \mathrm{~kg}^{-2 / 3}$ \\
\hline$\beta_{3}$ & $\bar{d}_{-}$ & & $3.46 \times 10^{2} \mathrm{~J}^{1 / 3} \mathrm{~m}^{-1}$ & $2.15 \mathrm{~kg}^{1 / 3} \mathrm{~m}^{-1}$ \\
\hline $\bar{d}_{3}$ & $d_{3}=\overline{\beta_{3} \ln 10}$ & & $1.25 \times 10^{-3} \mathrm{~m} \mathrm{~J}^{-1 / 3}$ & $0.202 \mathrm{~m} \mathrm{~kg}^{-1 / 3}$ \\
\hline$p_{\mathrm{a}, \mathrm{ref}}$ & & & $1.01325 \times 10^{5} \mathrm{~Pa}$ & $1.01325 \times 10^{5} \mathrm{~Pa}$ \\
\hline$E_{\mathrm{kg}, \mathrm{TNT}}$ & & & $4.184 \times 10^{6} \mathrm{~J}$ & $1 \mathrm{~kg}$ \\
\hline
\end{tabular}

\section{Appendix C Impulse Depth- and Distance Dependency}

Ford et al. (2014) found the following model to fit scaled blast impulse, distance and depth:

$$
\log _{10} \bar{I}=\beta_{1}+\log _{10} \bar{r}+\beta_{3} \bar{h}-\log _{10}\left(1+10^{10 \beta_{3} \bar{h}}\right) / 10
$$

Here $\bar{h}$ is the scaled height of burst, and energy was specified in kg TNT. Changing to scaled depth of explosion $(\bar{d}=-\bar{h})$, this can be written as

$$
\bar{I}_{1}=\frac{b_{1}}{\bar{r}} \frac{e^{-\bar{d} / \bar{d}_{3}}}{\left(1+e^{-10 \bar{d} / \bar{d}_{3}}\right)^{1 / 10}} .
$$

Constants $b_{1}$ and $\bar{d}_{3}$ are listed in Table C1. Ford et al. present the scaled impulse multiplied by ambient reference pressure, which is different from this study where scaled impulse is scaled overpressure integrated over energy-scaled time. We note that for $\bar{d}=$ $\bar{h}=0$ the depth dependent part reduces to $2^{-0.1} \simeq 0.93$, which is about $7 \%$ different from an exponential $\left(e^{0}=1\right)$. For larger depths this difference is smaller, which justifies the use of an exponential depth part (Appendix A) without the reducing factor which is necessary above the surface:

$$
\bar{I}=\frac{b_{1}}{\bar{r}} e^{-\bar{d} / \bar{d}_{3}}
$$




\section{Acknowledgments}

The authors acknowledge NSF grant EAR-1420455 for funding the necessary blasting resources, and University at Buffalo for hosting a workshop during which experiments were conducted. Kayley Diem-Kay, Norman Yu and David Hyman are acknowledged for their assistance with experiment preparation and data recording.

The authors express appreciation to Kent Gee, Dept. of Physics and Astronomy at Brigham Young University (BYU) for providing the equipment used for the broadband microphone measurements in this experiment. Funding for the BYU acoustical measurement team's participation came from the BYU College of Physical and Mathematical Sciences that funded six undergraduate research assistant: Sarah Ostergaard, Eric Lynsenko, Grace McKay Smith, Christian Lopez, Carla Wallace, and Menley Hawkes; and the NSF-funded research Experience for Teachers hosted at BYU that allowed middle school science teacher Julio Escobedo to play a pivotal role.

We thank Sean Maher and Richard Sanderson for their assistance with the seismic and infrasound field instrumentation preparation and deployment. Matoza acknowledges NSF grant EAR-1847736.

All measured data is hosted as datasets on VHub (vhub.org). A summarizing dataset which makes all data available is available at https://vhub.org/resources/4710. Data is also available separately as listed in the supporting information document (dataset S1).

\section{References}

Ahern, T. K., \& Dost, B. (2012, August). SEED Standard for the Exchange of Earthquake Data. (Reference Manual No. Format version 2.4.). Retrieved from https://www.fdsn.org/pdf/SEEDManual_V2.4.pdf

Ambrosini, R. D., \& Luccioni, B. M. (2006). Craters produced by explosions on the soil surface. Journal of Applied Mechanics, 73(6), 890. doi: 10.1115/1 .2173283

Ambrosini, R. D., Luccioni, B. M., Danesi, R. F., Riera, J. D., \& Rocha, M. M. (2002, July). Size of craters produced by explosive charges on or above the ground surface. Shock Waves, 12(1), 69-78. doi: 10.1007/s00193-002-0136-3

Antoun, T. H., Lomov, I. N., \& Glenn, L. A. (2003, December). Simulation of the penetration of a sequence of bombs into granitic rock. International Journal of Impact Engineering, 29(1-10), 81-94.

Arora, H., Del Linz, P., \& Dear, J. (2017, June). Damage and deformation in composite sandwich panels exposed to multiple and single explosive blasts. International Journal of Impact Engineering, 104, 95-106. doi: 10.1016/j.ijimpeng .2017 .01 .017

Boatwright, J. (1980). A spectral theory for circular seismic sources; simple estimates of source dimension, dynamic stress drop, and radiated seismic energy. Bulletin of the Seismological Society of America, 70(1), 1-27.

Bowman, D. C., Taddeucci, J., Kim, K., Anderson, J. F., Lees, J. M., Graettinger, A. H., ... Valentine, G. A. (2014). The acoustic signatures of ground acceleration, gas expansion, and spall fallback in experimental volcanic explosions. Geophysical Research Letters, 41(6), 1916-1922. doi: 10.1002/2014GL059324

Bush, V., Conant, J. P., \& Wilson, E. B. J. (1946). Effects of Impact and Explosion (Technical Report No. AD0221586). Washington DC: Office of Scientific Research and Development. Retrieved from http://www.dtic.mil/get-tr-doc/ pdf?AD=AD0221586

Büttner, R., \& Zimanowski, B. (1998). Physics of thermohydraulic explosions. Physical Review E, 57(5), 5726-5729. doi: 10.1103/PhysRevE.57.5726

Crighton, D. G., \& Scott, J. F. (1979, August). Asymptotic solutions of model equations in nonlinear acoustics. Philosophical Transactions of the Royal Society of 
London. Series A, Mathematical and Physical Sciences, 292(1389), 101-134. doi: $10.1098 /$ rsta.1979.0046

Dillon, L. A. (1972). The influence of soil and rock properties on the dimensions of explosion-produced craters (Technical Report No. AD0891964). New Mexico: Air Force Weapons Laboratory, Air Force Systems Command Kirtland Air Force Base. Retrieved from http://www.dtic.mil/docs/citations/ AD0891964

Dürig, T., Gudmundsson, M., \& Dellino, P. (2015). Reconstruction of the geometry of volcanic vents by trajectory tracking of fast ejecta - the case of the Eyjafjallajokull 2010 eruption (Iceland). Earth, Planets and Space, 67(1), 64. doi: 10.1186/s40623-015-0243-x

Dürig, T., Gudmundsson, M. T., Karmann, S., Zimanowski, B., Dellino, P., Rietze, M., \& Büttner, R. (2015). Mass eruption rates in pulsating eruptions estimated from video analysis of the gas thrust-buoyancy transition - a case study of the 2010 eruption of Eyjafjallajökull, Iceland. Earth, Planets and Space, 67(1), 1-17. doi: 10.1186/s40623-015-0351-7

Ehrgott, J., John Q., Akers, S. A., Windham, J. E., Rickman, D. D., \& Danielson, K. T. (2011). The influence of soil parameters on the impulse and airblast overpressure loading above surface-laid and shallow-buried explosives. Shock and Vibration, 18(6). doi: 10.3233/SAV-2010-0609

Fee, D., Waxler, R., Assink, J., Gitterman, Y., Given, J., Coyne, J., ... Grenard, P. (2013, June). Overview of the 2009 and 2011 Sayarim Infrasound Calibration Experiments: Sayarim Infrasound Overview. Journal of Geophysical Research: Atmospheres, 118(12), 6122-6143. doi: 10.1002/jgrd.50398

Ford, S. R., Rodgers, A. J., Xu, H., Templeton, D. C., Harben, P., Foxall, W., \& Reinke, R. E. (2014, March). Partitioning of seismoacoustic energy and estimation of yield and height-of-burst/depth-of-burial for near-surface explosions. Bulletin of the Seismological Society of America, 104(2), 608-623. doi: $10.1785 / 0120130130$

Garces, M. (2018, October). Explosion source models. In Infrasound monitoring for atmospheric studies (pp. 273-345). Springer International Publishing. doi: 10 .1007/978-3-319-75140-5_8

Garcés, M. A., Fee, D., \& Matoza, R. S. (2013). Modeling Volcanic Processes: The Physics and Mathematics of Volcanism (S. A. Fagents, T. K. P. Gregg, \& R. M. C. Lopes, Eds.). Cambridge: Cambridge University Press. doi: $10.1017 /$ CBO9781139021562

Gaudin, D., Taddeucci, J., Houghton, B. F., Orr, T. R., Andronico, D., Bello, E. D., ... Scarlato, P. (2016, October). 3-D high-speed imaging of volcanic bomb trajectory in basaltic explosive eruptions. Geochemistry, Geophysics, Geosystems, 17(10), 4268-4275. doi: $10.1002 / 2016 \mathrm{gc} 006560$

Gonnermann, H. M., \& Manga, M. (2007). The fluid mechanics inside a volcano. Annual Review of Fluid Mechanics, 39, 321-356. doi: 10.1146/annurev.fluid.39 .050905 .110207

Goto, A., Taniguchi, H., Yoshida, M., Ohba, T., \& Oshima, H. (2001). Effects of explosion energy and depth to the formation of blast wave and crater: Field Explosion Experiment for the understanding of volcanic explosion. Geophysical Research Letters, 28(22), 4287-4290. doi: 10.1029/2001GL013213

Grady, D. E. (1996). Shock-wave properties of brittle solids. In AIP Conference Proceedings (Vol. 370, pp. 9-20). Seattle, Washington (USA): AIP. doi: 10.1063/ 1.50579

Graettinger, A. H., Valentine, G. A., \& Sonder, I. (2015). Circum-crater variability of deposits from discrete, laterally and vertically migrating volcanic explosions: Experimental evidence and field implications. Journal of Volcanology and Geothermal Research, 308, 61-69. doi: 10.1016/j.jvolgeores.2015.10.019

Graettinger, A. H., Valentine, G. A., Sonder, I., Ross, P.-S., \& White, J. D. L. 
(2015). Facies distribution of ejecta in analog tephra rings from experiments with single and multiple subsurface explosions. Bulletin of Volcanology, 77(8), 1-12. doi: 10.1007/s00445-015-0951-x

Graettinger, A. H., Valentine, G. A., Sonder, I., Ross, P.-S., White, J. D. L., \& Taddeucci, J. (2014). Maar-diatreme geometry and deposits: Subsurface blast experiments with variable explosion depth. Geochem. Geophys. Geosys., 15(3), 740-764. doi: 10.1002/2013GC005198

Guzas, E. L., \& Earls, C. J. (2010, August). Air blast load generation for simulating structural response. Steel and Composite Structures, 10(5), 429-455.

Holsapple, K. A., \& Schmidt, R. M. (1980). On the scaling of crater dimensions: 1. Explosive processes. Journal of Geophysical Research, 85(B12), 7247-7256. doi: $10.1029 / \mathrm{JB} 085 \mathrm{iB} 12 \mathrm{p} 07247$

Houghton, B. (2015). Explosive volcanism. In H. Sigurdsson, B. Houghton, S. R. McNutt, H. Rymer, \& J. Stix (Eds.), The encyclopedia of volcanoes (Second ed., pp. 457-686). Elsevier LTD, Oxford.

Johnson, J., \& Aster, R. (2005, December). Relative partitioning of acoustic and seismic energy during Strombolian eruptions. Journal of Volcanology and Geothermal Research, 148(3-4), 334-354._doi: 10.1016/ j.jvolgeores.2005.05.002

Kim, K., \& Rodgers, A. (2016, July). Waveform inversion of acoustic waves for explosion yield estimation. Geophysical Research Letters, 43(13), 6883-6890. doi: $10.1002 / 2016 \mathrm{gl} 1069624$

Kim, K., \& Rodgers, A. (2017, August). Influence of low-altitude meteorological conditions on local infrasound propagation investigated by 3-D fullwaveform modeling. Geophysical Journal International, 210(2), 1252-1263. doi: $10.1093 / \mathrm{gji} / \operatorname{ggx} 218$

Kinney, G. F., \& Graham, K. J. (1985). Explosive shocks in air. Springer.

Lee, C. K. B., \& Mazzola, T. A. (1989). Ejecta scaling laws for craters in dry alluvial sites. Journal of Geophysical Research: Solid Earth, 94(B12), 1759517605. doi: $10.1029 / J B 094$ iB12p17595

Lorenz, V. (1975). Formation of phreatomagmatic maar-diatreme volcanoes and its relevance to kimberlite diatremes. Physics and Chemistry of the Earth, 9(0), 17-27. doi: 10.1016/0079-1946(75)90003-8

Macorps, É., Graettinger, A. H., Valentine, G. A., Ross, P.-S., White, J. D. L., \& Sonder, I. (2016, March). The effects of the host-substrate properties on maardiatreme volcanoes: Experimental evidence. Bulletin of Volcanology, 78(4). doi: $10.1007 / \mathrm{s} 00445-016-1013-8$

Maher, S. P., Matoza, R. S., Groot-Hedlin, C. D., Gee, K. L., Fee, D., \& Yokoo, A. (2020, March). Investigating Spectral Distortion of Local Volcano Infrasound by Nonlinear Propagation at Sakurajima Volcano, Japan. Journal of Geophysical Research: Solid Earth, 125(3). doi: 10.1029/2019JB018284

Mammadova, N., Ghaisas, S., Zenitsky, G., Sakaguchi, D. S., Kanthasamy, A. G., Greenlee, J. J., \& West Greenlee, M. H. (2017, July). Lasting Retinal Injury in a Mouse Model of Blast-Induced Trauma. The American Journal of Pathology, 187(7), 1459-1472. doi: 10.1016/j.ajpath.2017.03.005

Marchetti, E., Ripepe, M., Delle Donne, D., Genco, R., Finizola, A., \& Garaebiti, E. (2013, November). Blast waves from violent explosive activity at Yasur Volcano, Vanuatu. Geophysical Research Letters, 40(22), 5838-5843. doi: 10.1002/2013GL057900

Matoza, R. S., Arciniega-Ceballos, A., Sanderson, R. W., Mendo-Pérez, G., RosadoFuentes, A., \& Chouet, B. A. (2019, January). High-broadband seismoacoustic signature of vulcanian explosions at Popocatépetl volcano, Mexico. Geophysical Research Letters, 46 (1), 148-157. doi: 10.1029/2018gl080802

Matoza, R. S., Fee, D., \& Lopez, T. M. $\quad$ (2014, November). Acoustic Characterization of Explosion Complexity at Sakurajima, Karymsky, and Tungu- 
rahua Volcanoes. $\quad$ Seismological Research Letters, 85(6), 1187-1199. doi: $10.1785 / 0220140110$

Matoza, R. S., Garcés, M. A., Chouet, B. A., D'Auria, L., Hedlin, M. A. H., De Groot-Hedlin, C., \& Waite, G. P. (2009, April). The source of infrasound associated with long-period events at Mount St. Helens. Journal of Geophysical Research, 114(B4), B04305. doi: 10.1029/2008JB006128

Muhlestein, M. B., Gee, K. L., \& Macedone, J. H. (2012, March). Educational demonstration of a spherically propagating acoustic shock. The Journal of the Acoustical Society of America, 131(3), 2422-2430. doi: 10.1121/1.3676730

Park, I., Jolly, A., Matoza, R. S., Kennedy, B., Kilgour, G., Johnson, R., ... Cevuard, S. (2021, September). Seismo-acoustic characterisation of the 2018 Ambae (Manaro Voui) eruption, Vanuatu. Bulletin of Volcanology, 83(9), 60. doi: $10.1007 / \mathrm{s} 00445-021-01474-\mathrm{z}$

Pistolesi, M., Delle Donne, D., Pioli, L., Rosi, M., \& Ripepe, M. (2011). The 15 March 2007 explosive crisis at Stromboli volcano, Italy: Assessing physical parameters through a multidisciplinary approach. Research: Solid Earth, 116(B12). doi: 10.1029/2011JB008527

Qiu, X., Shi, X., Gou, Y., Zhou, J., Chen, H., \& Huo, X. (2018, April). Short-delay blasting with single free surface: Results of experimental tests. Tunnelling and Underground Space Technology, 74, 119-130. doi: 10.1016/j.tust.2018.01.014

Rogers, P. H. (1977). Weak-shock solution for underwater explosive shock waves. The Journal of the Acoustical Society of America, 62(6), 1412. doi: 10.1121/1 .381674

Ross, P.-S., White, J. D. L., Valentine, G. A., Taddeucci, J., Sonder, I., \& Andrews, R. G. (2013). Experimental birth of a maar-diatreme volcano. Journal of Volcanology and Geothermal Research, 260, 1-12. doi: 10.1016/j.jvolgeores.2013.05.005

Sato, H., \& Taniguchi, H. (1997). Relationship between crater size and ejecta volume of recent magmatic and phreato-magmatic eruptions: Implications for energy partitioning - range. Geophysical Research Letters, 24(3), 205-208. doi: 10.1029/96GL04004

Schnurr, J., Kim, K., Garces, M. A., \& Rodgers, A. (2020, May). Improved Parametric Models for Explosion Pressure Signals Derived From Large Datasets. Seismological Research Letters, 91 (3), 1752-1762. doi: 10.1785/0220190278

Sonder, I., Graettinger, A. H., \& Valentine, G. A. (2015). Scaling multiblast craters: General approach and application to volcanic craters. Journal of Geophysical Research, Solid Earth, 120(9), 6141-6158. doi: 10.1002/2015JB012018

Strange, J. N., Denzel, C. W., \& McLane, T. I., III. (1960). Cratering from high explosive charges. Analysis of crater data (Tech. Rep. No. AD0263170). Vicksburg, MS: Army Engineer Waterways Experiment Station. Retrieved from http://oai.dtic.mil/oai/oai?verb=getRecord\&metadataPrefix= html\&identifier $=$ AD0263170

Taddeucci, J., Peña Fernández, J. J., Cigala, V., Kueppers, U., Scarlato, P., Del Bello, E., ... Panunzi, S. (2021, August). Volcanic Vortex Rings: Axial Dynamics, Acoustic Features, and Their Link to Vent Diameter and Supersonic Jet Flow. Geophysical Research Letters, 48(15). doi: 10.1029/2021GL092899

Taddeucci, J., Sottili, G., Palladino, D., Ventura, G., \& Scarlato, P. (2010). A note on maar eruption energetics: Current models and their application. Bulletin of Volcanology, 72 (1), 75-83. doi: 10.1007/s00445-009-0298-2

Taylor, Z. J., Gurka, R., Kopp, G. A., \& Liberzon, A. (2010). Long-duration timeresolved PIV to study unsteady aerodynamics. IEEE $E_{J I} M, 59(12), 3262-3269$. doi: 10.1109/TIM.2010.2047149

Valentine, G. A., Graettinger, A. H., Macorps, É., Ross, P.-S., White, J. D. L., Döhring, É., \& Sonder, I. (2015). Experiments with vertically and lat- 
erally migrating subsurface explosions with applications to the geology of phreatomagmatic and hydrothermal explosion craters and diatremes. Bulletin of Volcanology, $77(3)$. doi: 10.1007/s00445-015-0901-7

Valentine, G. A., Graettinger, A. H., \& Sonder, I. (2014). Explosion depths for phreatomagmatic eruptions. Geophysical Research Letters, 41(9), 3045-3051. doi: 10.1002/2014GL060096

Valentine, G. A., White, J. D. L., Ross, P.-S., Amin, J., Taddeucci, J., Sonder, I., \& Johnson, P. J. (2012). Experimental craters formed by single and multiple buried explosions and implications for maar-diatreme volcanoes. Geophysical Research Letters, 39, L20301. doi: 10.1029/2012GL053716

Voight, B. (1981). Time scale for the first moments of the May 18 eruption. In P. W. Lipman \& D. R. Mullineaux (Eds.), The 1980 eruptions of mount st. Helens, washington (Vol. 1250, pp. 69-93). U.S. Government Printing Office.

Vortman, L. J. (1968). Craters from surface explosions and scaling laws. Journal of Geophysical Research, 73(14), 4621-4636. doi: 10.1029/JB073i014p04621

Young, S. M., Gee, K. L., Neilsen, T. B., \& Leete, K. M. (2015, September). Outdoor measurements of spherical acoustic shock decay. The Journal of the Acoustical Society of America, 138(3), EL305-EL310. doi: 10.1121/1.4929928

Zhou, J., Lu, W., Yan, P., Chen, M., \& Wang, G. (2016, October). FrequencyDependent Attenuation of Blasting Vibration Waves. Rock Mechanics and Rock Engineering, 49(10), 4061-4072. doi: 10.1007/s00603-016-1046-5 